\title{
Upstream swimming and Taylor dispersion of active Brownian particles
}

\author{
Zhiwei Peng $\odot$ and John F. Brady* \\ Division of Chemistry and Chemical Engineering, California Institute of Technology, \\ Pasadena, California 91125, USA
}

(Received 10 October 2019; accepted 1 June 2020; published 8 July 2020)

\begin{abstract}
Locomotion of self-propelled particles such as motile bacteria or phoretic swimmers often takes place in the presence of applied flows and confining boundaries. Interactions of these active swimmers with the flow environment are important for the understanding of many biological processes, including infection by motile bacteria and the formation of biofilms. Recent experimental and theoretical works have shown that active particles in a Poiseuille flow exhibit interesting dynamics including accumulation at the wall and upstream swimming. Compared to the well-studied Taylor dispersion of passive Brownian particles, a theoretical understanding of the transport of active Brownian particles (ABPs) in a pressure-driven flow is relatively less developed. In this paper, employing a small wave-number expansion of the Smoluchowski equation describing the particle distribution, we explicitly derive an effective advection-diffusion equation for the cross-sectional average of the particle number density in Fourier space. We characterize the average drift (specifically upstream swimming) and effective longitudinal dispersion coefficient of active particles in relation to the flow speed, the intrinsic swimming speed of the active particles, their Brownian diffusion, and the degree of confinement. In contrast to passive Brownian particles, both the average drift and the longitudinal dispersivity of ABPs exhibit a nonmonotonic variation as a function of the flow speed. In particular, the dispersion of ABPs includes the classical shear-enhanced (Taylor) dispersion and an active contribution called the swim diffusivity. In the absence of translational diffusion, the classical Taylor dispersion is absent and we observe a giant longitudinal dispersion in the strong flow limit. Our continuum theory is corroborated by a direct Brownian dynamics simulation of the Langevin equations governing the motion of each ABP.
\end{abstract}

DOI: 10.1103/PhysRevFluids.5.073102

\section{INTRODUCTION}

Transport and mixing within suspensions of swimming micro-organisms are important for the understanding of many biological and industrial processes, such as infection by motile bacteria [1,2] or the formation of biofilms [3,4]. Different from passive particles, the ability of active swimmers to self-propel gives rise to interesting dynamics including accumulation at confining boundaries and upstream swimming in the presence of a pressure-driven flow [5].

The transport of passive matter (e.g., diffusing solute) that experiences molecular diffusion and fluid advection in a Poiseuille flow has been extensively studied since the seminal work of Taylor [6-8], who demonstrated that the effective dispersion coefficient in the direction of flow is enhanced compared to the bare molecular diffusivity. Shortly after, Aris [9] underpinned Taylor's analysis with a theoretical framework based on longitudinal moments of the solute distribution function. This phenomenon of enhanced longitudinal spreading, often referred to as Taylor (or Taylor-Aris)

\footnotetext{
*jfbrady@caltech.edu
} 


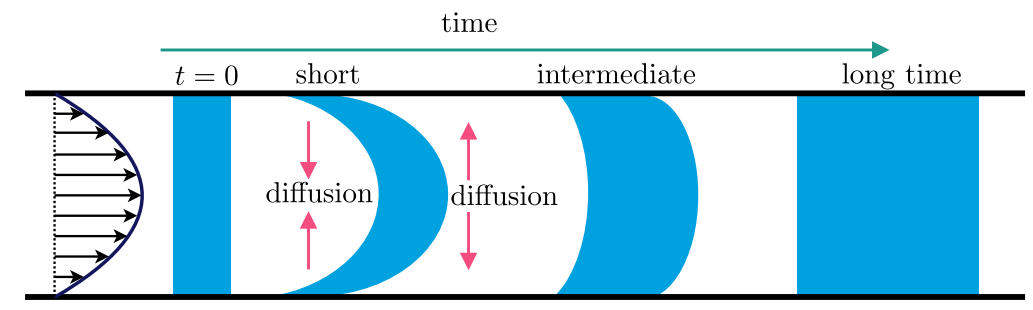

FIG. 1. Schematic of the Taylor dispersion process of passive matter in a pressure driven flow.

dispersion, results from the coupling of molecular diffusion in the transverse direction and advection in the longitudinal direction. To understand the interaction between these two separate mechanisms for solute transport, it is helpful to first consider advection alone, and then to include the effect of molecular diffusion. In the absence of molecular diffusion, a point-sized particle introduced into the flow simply moves downstream along the streamline at that location. The presence of molecular diffusion, on the other hand, enables the particle to migrate across streamlines and then immediately be advected downstream with different velocities. This process represents a random walk and leads to an advective contribution to the longitudinal dispersion in addition to the molecular diffusivity. In a random-walk process, the diffusivity scales as $l^{2} / \tau$, where $l$ is the step size and $\tau$ is the decorrelation time. Consider a Brownian solute with diffusivity $D_{T}$ in a pressure-driven channel flow with characteristic width $H$ and centerline speed $u_{0}$. The decorrelation time is purely Brownian and given by $\tau \sim H^{2} / D_{T}$ while the step size is due to advection in this time: $l \sim u_{0} \tau$. As a result, we have $\left(D^{\text {eff }}-D_{T}\right) / D_{T} \sim \mathrm{Pe}^{2}$, where $D^{\text {eff }}$ is the effective longitudinal dispersivity and $\mathrm{Pe}=u_{0} H / D_{T}$ is the Péclet number.

One can also rationalize the classical Taylor dispersion from a macroscopic point of view. Suppose that initially a diffusing solute is introduced at a small segment of the channel flow with a uniform concentration (see Fig. 1 for a schematic). At times shorter than the diffusive time scale $H^{2} / D_{T}$, the nonuniform fluid advection generates a concentration gradient across the channel, which induces a diffusive flux of the material transverse to the direction of flow. At long times, the concentration in the transverse direction becomes uniform again due to molecular diffusion. In this long-time asymptotic limit, the cross-sectional average concentration is governed by an effective one-dimensional advection-diffusion equation. The drift velocity in this equation is simply the cross-sectional average fluid velocity, while the effective Taylor dispersivity is greater than the molecular diffusivity.

During the past half century, the original analysis by Taylor and Aris has been extended to accommodate a wide class of dispersion problems including complex geometries $[10,11]$ and chemical reactions [12,13]. In particular, a generalized Taylor dispersion theory (GTDT) has been developed [14]. GTDT provides a systematic scheme to derive the coarse-grained transport equation starting from the probability density function describing the motion of a Brownian tracer in its phase space. A detailed discussion on the application of GTDT to material dispersion can be found in the book by Brenner and Edwards [15].

In contrast to passive tracers, the study of the transport of active microswimmers that self-propel is more recent and their dynamics is less well understood. To capture the essential physics of a microswimmer on a coarse-grained level, the active Brownian particle (ABP) model is often used (see Fig. 2). An ABP self-propels with a fixed intrinsic speed $U_{0}$ and undergoes translational and rotational Brownian motion with diffusivities $D_{T}$ and $D_{R}$. We note that in modeling the locomotion of swimming microorganisms the diffusivities are typically biological rather than thermal in origin. The rotary diffusion describes the random reorientation of microorganisms such as tumbling. The translational diffusivity can be interpreted as a simple model representing the fluctuation of the center-of-mass position during the flagellar bundling or unbundling process. In other words, $D_{T}$ and $D_{R}$ are independent quantities and not constrained by the fluctuation-dissipation theorem. Due to 

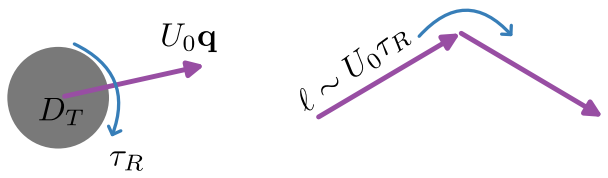

active random walk

$D^{\mathrm{swim}} \sim \frac{\ell^{2}}{\tau_{R}} \sim U_{0}^{2} \tau_{R}$

FIG. 2. Schematic of an ABP in an unbounded domain with no background flow and its long-time $\left(t \gg \tau_{R}\right)$ active random-walk process. The swimming direction is denoted by a unit vector $\mathbf{q}$.

rotary Brownian motion, the swimming direction of an $\mathrm{ABP}$ relaxes over the reorientation time scale $\tau_{R}=1 / D_{R}$. One can define a run length $\ell=U_{0} \tau_{R}$ that characterizes the persistence of swimming in a given direction. The directed swimming persists at short time $\left(t \ll \tau_{R}\right)$ and one observes ballistic motion. At times much larger than $\tau_{R}$, the swimming motion becomes a random walk due to Brownian reorientation. This active random walk can be characterized by an effective diffusivity $D^{\text {swim }} \sim \ell^{2} / \tau_{R} \sim U_{0}^{2} \tau_{R}$ called the swim diffusivity. A detailed calculation gives $D^{\text {swim }}=U_{0}^{2} \tau_{R} / 2$ in two-dimensional unbounded space [16]. This implies that the long-time dispersion coefficient of ABPs is enhanced, $D^{\text {eff }}=D_{T}+D^{\text {swim }}[17,18]$. The swim-enhanced dispersion originates from the coupling of diffusion in orientational space to the translational via self-propulsion, which is a unique signature of active matter systems.

When placed under confinement, such as in a channel, ABPs tend to accumulate at the wall. Unlike noninteracting Brownian particles in thermal equilibrium that exhibits a uniform density distribution, ABPs can spontaneously generate regions of higher density under geometric confinement. This boundary accumulation is another distinct feature of active matter, which is ubiquitous in biological processes involving motile bacteria or swimming spermatozoa. Exploiting this behavior, Sokolov et al. [19] showed that a motile bacteria suspension can power microscopic gears with asymmetric teeth. Interestingly, this boundary accumulation phenomenon was first observed decades ago by Rothschild [20], who measured a nonuniform concentration of swimming bull spermatozoa in a glass chamber. More recently, experiments by Berke et al. [21] reported the same accumulation phenomenon with swimming Escherichia coli (E. coli) confined between two glass plates. Rothschild postulated that wall accumulation might originate from hydrodynamic interaction of swimmers with the wall. Berke et al. pursued this idea and provided a theoretical model to explain their experimental results. On the other hand, studies neglecting hydrodynamic interaction between swimmers and boundary also observed wall accumulation [22-27]. These studies suggest that wall accumulation can be understood from a purely kinematic perspective while hydrodynamic interaction only modifies this behavior quantitatively. That is, owing to persistent self-propulsion, a swimmer hitting the boundary maintains its orientation for a finite time $\tau_{R}$ until it rotates away via Brownian reorientation. Conversely, swimmers pointing away from the boundary simply swim into the bulk. As a result, there is an accumulation of swimmers near the boundary with a net orientation or polar order pointing into the boundary.

To gain a quantitative understanding, consider ABPs confined between two parallel plates separated by a distance $H$. Before considering the number density and polar order distribution, it is helpful to recognize the three length scales in this problem: the run length $\ell$, a microscopic length $\delta=\sqrt{D_{T} \tau_{R}}$ that characterizes the distance an ABP travels by diffusion within the reorientation time, and the channel width $H$. Two dimensionless groups, $\ell / H$ and $\ell / \delta$, can thus be defined. The dimensionless quantity $\ell / \delta$ compares the distance traveled by swimming to translational diffusion in $\tau_{R}$, and thus characterizes the activity level of ABPs. The second parameter $\ell / H$ quantifies the strength of confinement. In the limit $\ell / H \ll 1$, an ABP can travel its full run length without colliding with the wall. If $\ell / H \gg 1$, the swimming trajectories of ABPs are frequently disrupted by the wall. We use orientational moments, in particular number density $\left(n^{0}\right)$ and polar order in the transverse direction $\left(m_{y}^{0}\right)$ as a function of the transverse coordinate $y$, to quantify the effect of confinement. The polar order $m_{y}^{0}$ characterizes the amount of particles pointing in the $\pm y$ direction (see Sec. II for the mathematical definition and formulation). Using a vanishing nematic order 

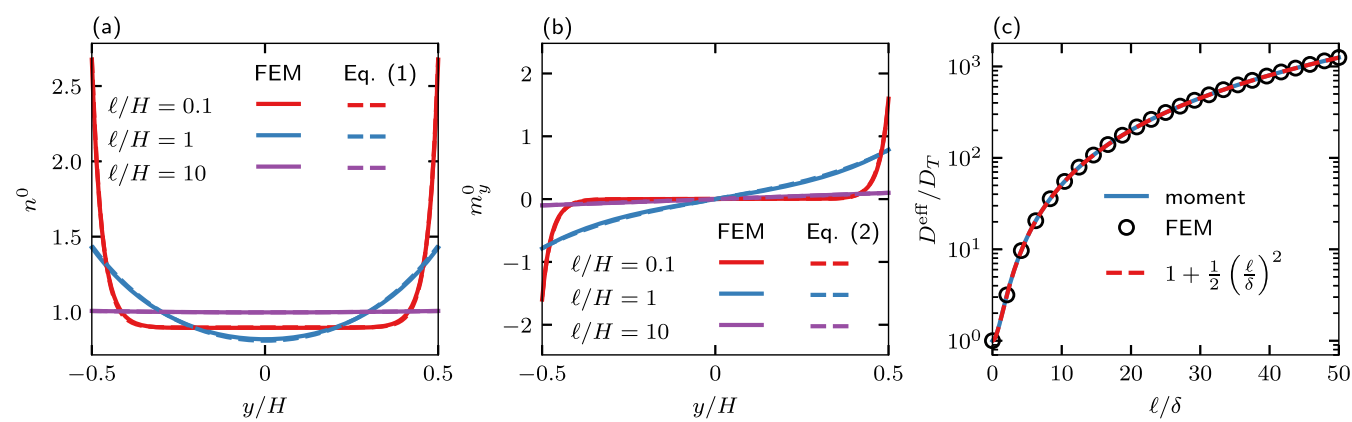

FIG. 3. (a) Number density $n^{0}$ as a function of the transverse coordinate $y$ for $\ell / \delta=U_{0} \tau_{R} / \sqrt{D_{T} \tau_{R}}=2$ and different confinement strengths: $\ell / H=U_{0} \tau_{R} / H=\{0.1,1,10\}$. (b) Polar order in the transverse direction $m_{y}^{0}$ as a function of the transverse coordinate $y$ for $\ell / \delta=2$ and different confinement strengths. (c) Effective longitudinal dispersion coefficient $D^{\text {eff }} / D_{T}$ as a function of activity $\ell / \delta$. Circles are results from finite element simulations and the solid line is the solution from moment equations. The free space effective dispersivity of ABPs is denoted by the dashed line. In all three panels, the flow is absent. Refer to Sec. II for details on FEM and moment equations.

closure to the Smoluchowski equation, Yan and Brady [28] obtained the number density and polar order distribution of ABPs in several confinement geometries. For ABPs between two parallel plates in two dimensions, it is shown that

$$
\begin{gathered}
\frac{n^{0}(y)}{\bar{n}}=1+\frac{1}{2}\left(\frac{\ell}{\delta}\right)^{2} \frac{\cosh (\lambda y)}{\cosh (\lambda H / 2)}, \\
\frac{m_{y}^{0}(y)}{\bar{n}}=\frac{1}{2} \lambda \ell \frac{\sinh (\lambda y)}{\cosh (\lambda H / 2)} .
\end{gathered}
$$

Here, $y \in[-H / 2, H / 2]$ is the transverse coordinate, $n^{0}$ is the number density, $m_{y}^{0}$ is the polar order in the transverse direction, and $\lambda=\sqrt{1+\frac{1}{2}(\ell / \delta)^{2}} / \delta$ is the inverse screening length. To be consistent with notation in this paper, the average concentration $\bar{n}$ is obtained such that $(1 / H) \int_{-H / 2}^{H / 2} n^{0} d y=1$. To prevent ABPs from penetrating the wall, the swimming flux into the wall $\left(U_{0} m_{y}^{0}\right)$ is balanced by a diffusive flux $\left(-D_{T} d n^{0} / d y\right)$ away from the wall [see Eq. (A23) in Appendix A]. This balance dictates that the screen length $\lambda^{-1}$ is proportional to the microscopic length $\delta$ and depends on the activity $\ell / \delta$. For a given confinement strength $(\ell / H)$, the accumulation boundary layer becomes thinner as activity $(\ell / \delta)$ increases. Since $\delta / H=\ell / H(\ell / \delta)^{-1}$, the boundary layers becomes thinner as confinement $\ell / H$ decreases for a fixed $\ell / \delta$. Therefore, we observe a sharp accumulation boundary layer for $\ell / H \ll 1$. As $\ell / H$ increases, the two boundary layers at the top and bottom walls merge and a more uniform number density profile is observed. The variation of the number density profile as a function of $\ell / H$ is shown in Fig. 3(a). Strong accumulation of $\mathrm{ABPs}$ at the wall is accompanied by a net polar order as seen in Fig. 3(b) for $\ell / H=0.1$. The case of passive Brownian particles, which has a uniform number density, can be obtained by setting $\ell=0$.

Due to the linear geometry of the channel, we can obtain the effective longitudinal dispersion coefficient by a simple physical argument. Because the Brownian kicks in each direction are independent, the confinement in the transverse direction does not affect motion in the longitudinal direction. We expect the effective longitudinal dispersion to be identical to that in free space, $D^{\text {eff }} / D_{T}=1+\frac{1}{2}(\ell / \delta)^{2}$. In Fig. 3(c), we show $D^{\text {eff }} / D_{T}$ as a function of $\ell / \delta$ obtained from both finite element simulations (circles) and moment equations (blue solid line). 
These results agree well with the free space dispersivity given by the red dashed line. We remark that confinement can have an effect on longitudinal dispersion if the channel width is nonuniform.

The application of an external flow in the channel further complicates the transport process of ABPs. First, ABPs are now advected by the flow in addition to its self-advection. Second, the orientational dynamics of ABPs are coupled to the local fluid shear, which varies spatially in a quadratic flow. One manifestation of such a coupling effect is the upstream swimming of motile particles in a pressure-driven flow. Here, we are speaking in terms of upstream swimming in a laboratory-fixed frame rather than that relative to the downstream flow. Put differently, a swimmer can overcome the downstream advection with some excess speed to move upstream. Koser and coworkers $[29,30]$ observed upstream swimming with $E$. coli in a microfluidic channel flow and proposed a mechanism for upstream motility involving chirality of the flagella and hydrodynamic interaction with the wall. A later study by Kaya and Koser [31] systematically measured the swim speed of E. coli and reported a transition between upstream and downstream motion. Below a critical but nonzero flow speed, E. coli were found to swim upstream. For strong flow, the swimming motion is dominated by fluid advection and net downstream motion is observed. In a recent work by Kantsler et al. [32], mammalian sperm cells are also found to exhibit upstream motility.

Various theoretical models have been proposed to explain the swimming dynamics of microorganisms in flow, many of which include the effect of fluid shear, body shape asymmetry, flagellar chirality, steric and/or hydrodynamic interaction with the wall, and so forth [24,3238]. These models are able to produce, at least qualitatively, upstream motion similar to those observed in experiments. On the other hand, Ezhilan and Saintillan [36] implemented a simpler model, which consists of noninteracting slender ABPs, to investigate the effect of flow on transport of microswimmers. Using numerical and asymptotic solutions to the Smoluchowski equation governing particle conservation, they investigated the distribution of ABPs across the channel systematically and predicted net upstream swimming. In their study, the focus is on mildly active ABPs that are moderately confined: $\ell / \delta=\sqrt{6}(\approx 2.45)$ and $\ell / H \leqslant 2$.

Previous work on the dispersion of active particles in flow has examined suspensions of gyrotactic micro-organisms [39-44] and chemotactic run-and-tumble bacteria [45]. Due to asymmetry in mass distribution, gyrotactic swimmers experience a gravitational torque in addition to the viscous torque from the local fluid vorticity. The balance between viscous and gravitational torques leads to a preferred orientation of the particles and thus a nonuniform particle distribution across the channel. More recently, Chilukuri et al. [46] investigated the dispersion of flagellated swimming micro-organisms in planar Poiseuille flow using Brownian dynamics (BD) simulation. In their work, a flagellated swimmer is represented by two beads connected by a stiff spring. They focused on the effect of flow speed on the dispersion of very active particles under moderate to strong confinement with $\ell / \delta=80$ and $\ell / H=\{2.5,4,10\}$. It is shown that the longitudinal dispersivity exhibits a nonmonotonic variation as a function of the flow speed with a minimum obtained when the flow speed is comparable to the self-propulsion speed. Intuitive physical scaling arguments were used to qualitatively explain the dispersion behavior in the weak and strong flow limits. Interestingly, a similar nonmonotonic dispersion behavior is predicted by Alonso-Matilla et al. [47] in the context of ABPs in external flow through periodic porous media. Dehkharghani et al. [48] studied the dispersion of swimming bacteria in flow through a microfluidic lattice consisting of a periodic array of circular pillars using both experiments and computer simulation. They showed that the external flow hinders transverse dispersion and enhances longitudinal dispersion of active particles with no translational diffusion beyond the Taylor dispersion of passive Brownian particles. Using the same ellipsoidal ABP model as Ezhilan and Saintillan [36], Jiang and Chen [49] studied the dispersion of active particles in confined unidirectional flows. The longitudinal dispersion of ABPs is related to the physical space moments of the probability density function of an ABP based on the GTDT. In this paper, they solved the resulting equations numerically and presented the dispersivity for $\ell / \delta=\sqrt{6}$ and $\ell / H=1$. 
In view of the above discussion, it is clear that upstream swimming is almost certainly universal to active particles and transcends some of the details of the specific mathematical model or microorganism used. This suggests an underlying mechanism shared among different models that is fundamental and responsible for upstream swimming, while additional details represent only a correction for the quantitative behavior. Though the dispersion of active particles in flow has been studied much less compared to upstream swimming, the same argument should apply. Indeed, as an example, Chilukuri et al. [46] showed that hydrodynamic interaction with the wall does not qualitatively alter the longitudinal dispersion of their beads-spring swimmers. Therefore, we believe that there is value in studying the dynamics of active particles in flow with a model that is simple yet able to capture the interesting advective-diffusive behavior discussed above. To this end, we consider spherical ABPs suspended in a Poiseuille flow between two parallel plates in two dimensions. Our paper ignores hydrodynamic interaction with the wall. In Appendix $C$, we discuss the effect of nonspherical shape that might be relevant to microorganisms such as E. coli. We also assume that the particle suspension is dilute so that the background flow is not affected by the suspended ABPs and excluded-volume interaction between particles is irrelevant.

In this paper, we show that both upstream swimming and nonmonotonic dispersion as a function of the flow speed are recovered using the spherical ABP model. Upstream swimming can be understood as a result of channel confinement and rotation by the flow alone. Particles at the wall are rotated towards the upstream direction owing to the fluid vorticity that reaches its maximum at the wall. Once aligned against the flow, self-propelled particles are able to swim upstream because the fluid velocity vanishes at the wall. Furthermore, ABPs accumulate at the wall when the flow is weak compared to self-propulsion. In the weak flow regime, strong upstream swimming is observed. As the flow speed increases, the intrinsic swim speed becomes subdominant and the number density of ABPs becomes more uniform across the channel. This leads to a transition from net upstream motion to downstream motion as the flow speed increases. The net speed of ABPs in the longitudinal direction approaches that of passive Brownian particles in the strong flow limit.

As predicted by previous work, the long-time longitudinal dispersion coefficient of ABPs is a nonmonotonic function of the flow speed. In addition to translational diffusion enjoyed by both active and passive Brownian particles, ABPs can also sample different flow speeds using self-propulsion coupled to a reorientation mechanism. For ABPs in flow, there are two mechanisms for reorientation: random rotary diffusion and deterministic rotation by the fluid vorticity. The longitudinal dispersion of ABPs consists of the translational diffusivity, the swim diffusivity, and the classical Taylor dispersivity. The swim diffusivity of ABPs is altered by the pressure-driven flow and the classical Taylor dispersivity of ABPs is different from that of passive Brownian particles because activity modifies the number density distribution. In the limit of weak flow, where ABPs explore the physical space dominantly via self-propulsion and random Brownian reorientation, the dispersion coefficient approaches that in the absence of flow. In the limit of strong flow, the dispersion coefficient converges to that of passive Brownian particles because ABPs lose their persistence due to the rapid rotation by the fluid vorticity.

We formulate the problem from a continuum perspective using the Smoluchowski equation governing the position and orientation of an ABP in the channel. We consider a dilute suspension and neglect particle-particle and particle-wall hydrodynamic interactions. From the Smoluchowski equation we derive an effective advection-diffusion equation for the cross-sectional average number density in Fourier space and calculate both the average drift and the effective dispersion coefficient in the flow direction in the long-time limit. In addition to the continuum model, the equivalent particle-based Langevin equations are considered. After the derivation of the governing equations, we elucidate the origin of upstream swimming by considering the case of deterministic particle dynamics without diffusion $\left(D_{T}=0\right.$ and $\left.D_{R}=0\right)$. We then characterize the drift velocity of active particles without translational diffusion $\left(D_{T}=0\right)$, followed by the general case of finite translational and rotational diffusion. Next, in Sec. IV we consider the dispersive behavior of active particles before concluding the paper in Sec. V. 


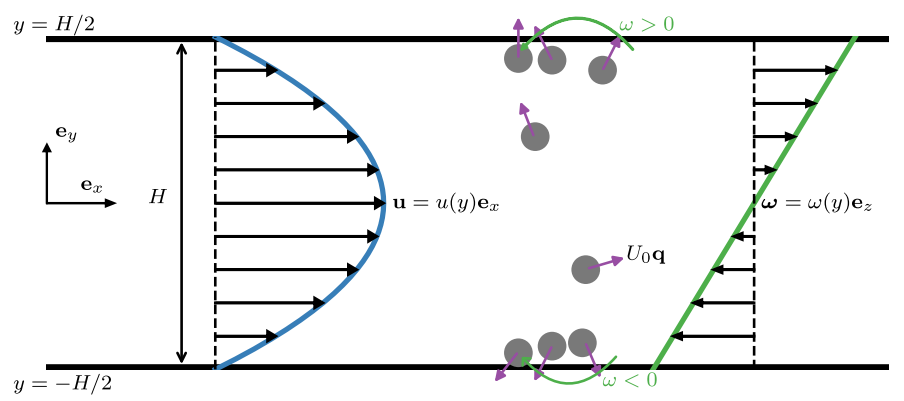

FIG. 4. Schematic of active Brownian particles suspended in a planar Poiseuille flow between two parallel plates. The ABPs reorient on a time scale of $\tau_{R}=1 / D_{R}$, where $D_{R}$ is the rotary diffusivity. The blue solid line is the Poiseuille flow and the green solid line is the scalar vorticity. The arrows represent the magnitude and sign of the fluid speed or vorticity. The vorticity is an odd function of the transverse coordinate $y$.

\section{PROBLEM FORMULATION}

We consider a dilute suspension of spherical ABPs in a planar Poiseuille flow between two parallel plates separated by a distance $H$ as illustrated in Fig. 4. We assume that the radius of the ABPs is negligible compared to the width of the channel. The background flow is given by $\mathbf{u}=u(y) \mathbf{e}_{x}$, where

$$
u=u_{0}\left(1-4 \frac{y^{2}}{H^{2}}\right), \quad-\frac{H}{2} \leqslant y \leqslant \frac{H}{2},
$$

with the maximum velocity $u_{0}$ at the centerline of the channel $(y=0)$.

Following previous work on active suspensions [50], we define a probability density function $P(\mathbf{x}, \mathbf{q}, t)$ of finding an ABP at position $\mathbf{x}$ with orientation $\mathbf{q}(|\mathbf{q}|=1)$ at time $t$. The evolution of $P$ satisfies the Smoluchowski equation:

$$
\frac{\partial P}{\partial t}+\nabla \cdot \mathbf{j}^{T}+\nabla_{R} \cdot \mathbf{j}^{R}=0
$$

where $\nabla$ and $\nabla_{R}=\mathbf{q} \times \frac{\partial}{\partial \mathbf{q}}$ are gradient operators in physical and orientation space, respectively. The translational and rotational fluxes are given by, respectively,

$$
\begin{gathered}
\mathbf{j}^{T}=\mathbf{u} P+U_{0} \mathbf{q} P-D_{T} \nabla P, \\
\mathbf{j}^{R}=\frac{1}{2} \boldsymbol{\omega} P-D_{R} \nabla_{R} P,
\end{gathered}
$$

where $\omega=\nabla \times \mathbf{u}$ is the vorticity vector of the background flow. The flux normal to a boundary surface must vanish, $\mathbf{e}_{y} \cdot \mathbf{j}^{T}=0$ at $y= \pm H / 2$. Integrating the Smoluchowski equation in orientation space gives a conservation equation for the number density:

$$
\frac{\partial n}{\partial t}+\nabla \cdot\left(\mathbf{u} n+U_{0} \mathbf{m}-D_{T} \nabla n\right)=0
$$

where $n(\mathbf{x}, t)=\int P d \mathbf{q}$ is the number density and $\mathbf{m}(\mathbf{x}, t)=\int \mathbf{q} P d \mathbf{q}$ is the polar order field.

The unboundedness in the flow direction makes it convenient to work in Fourier space. To this end, we introduce a Fourier transform with respect to $x$ given by $\hat{f}(k)=\int \exp (-i k x) f(x) d x$. In Fourier space, the governing equation for number density becomes

$$
\frac{\partial \hat{n}}{\partial t}+i k\left(u \hat{n}+U_{0} \hat{m}_{x}\right)-D_{T}(i k)^{2} \hat{n}+\frac{\partial}{\partial y}\left(U_{0} \hat{m}_{y}-D_{T} \frac{\partial \hat{n}}{\partial y}\right)=0 .
$$


Averaging over the width of the channel, we obtain

$$
\frac{\partial \bar{n}}{\partial t}+k^{2} D_{T} \bar{n}+i k\left(\overline{u(y) \hat{n}}+U_{0} \overline{\hat{m}}_{x}\right)=0
$$

where the boundary terms vanish due to the zero normal flux condition and an overhead bar denotes cross-sectional average, $\bar{n}(k, t)=(1 / H) \int_{-H / 2}^{H / 2} \hat{n}(k, y, t) d y$. It is convenient to relate $\hat{P}$ to $\bar{n}$ via the definition of a structure function $\hat{g}$ such that

$$
\hat{P}(k, y, \mathbf{q}, t)=\bar{n}(k, t) \hat{g}(k, y, \mathbf{q}, t) .
$$

By construction, we have the normalization condition

$$
\frac{1}{H} \int d y \int \hat{g} d \mathbf{q}=1 \text {. }
$$

On the other hand, taking the Fourier transform of the Smoluchowski equation (4) yields

$$
\frac{\partial \hat{P}}{\partial t}+i k \hat{j}_{x}^{T}+\frac{\partial \hat{j}_{y}^{T}}{\partial y}+\nabla_{R} \cdot \hat{\mathbf{j}}^{R}=0 .
$$

Using the definition of $\hat{P}$ given in Eq. (10), we multiply Eq. (9) by $\hat{g}$ and subtract it from Eq. (12) to obtain

$$
\begin{gathered}
\frac{\partial \hat{g}}{\partial t}+\frac{\partial}{\partial y}\left(U_{0} q_{y} \hat{g}-D_{T} \frac{\partial \hat{g}}{\partial y}\right)-D_{R} \nabla_{R}^{2} \hat{g}+\nabla_{R} \cdot\left(\frac{1}{2} \omega \hat{g}\right) \\
-i k\left(U_{0} \overline{\hat{m}}_{x} \frac{\hat{g}}{\bar{n}}+\overline{u \hat{n}} \frac{\hat{g}}{\bar{n}}-U_{0} q_{x} \hat{g}-u \hat{g}\right)=0 .
\end{gathered}
$$

Consider a small wave-number expansion, $\hat{g}=g_{0}(y, \mathbf{q}, t)+i k d_{0}(y, \mathbf{q}, t)+O\left(k^{2}\right)$, corresponding to a slow variation over a large length scale (weak gradient) [18,51]. Substituting this expansion into Eq. (9), we obtain an effective advection-diffusion equation for the average number density in Fourier space:

$$
\frac{\partial \bar{n}}{\partial t}+i k U^{\mathrm{eff}} \bar{n}+k^{2} D^{\mathrm{eff}} \bar{n}=0
$$

where the average drift and the effective longitudinal dispersion coefficient are

$$
\begin{gathered}
U^{\mathrm{eff}}=U_{0} \overline{m_{x}^{0}}+\overline{u(y) n^{0}}, \\
D^{\mathrm{eff}}=D_{T}-U_{0} \overline{\tilde{m}_{x}}-\overline{u(y) \tilde{n}} .
\end{gathered}
$$

Here, the average and fluctuating field variables are defined as the orientational moments of $g_{0}$ and $d_{0}$ :

$$
n^{0}=\int g_{0} d \mathbf{q}, \quad \mathbf{m}^{0}=\int \mathbf{q} g_{0} d \mathbf{q}, \quad \tilde{n}=\int d_{0} d \mathbf{q}, \quad \tilde{\mathbf{m}}=\int \mathbf{q} d_{0} d \mathbf{q} .
$$

The average drift of ABPs consists of the average of the flow speed weighted by the number density, $\overline{u(y) n^{0}}$, and the average streamwise swimming velocity $U_{0} \overline{m_{x}^{0}}$. Similarly, the effective longitudinal dispersion coefficient includes the translational diffusion $D_{T}$, the fluctuation in the self-propulsive velocity, $-U_{0} \overline{\tilde{m}_{x}}$, and the fluctuation in the particle number density weighted by the flow, $-\overline{u(y) \tilde{n}}$.

The classical Taylor dispersion of passive Brownian particles can be readily recovered by setting $U_{0}=0$. Without self-propulsion, the rotary diffusion is decoupled from the translational motion (and therefore is irrelevant) and the number density becomes uniform, $n^{0} \equiv 1$. As a result, we obtain $U^{\text {eff }}=\bar{u}=2 u_{0} / 3$ and $D^{\text {eff }}=D_{T}-\overline{u \tilde{n}}$ for passive particles. In contrast, the ability of ABPs to selfpropel alters the number density distribution and introduces additional terms in the expressions for the average drift and the effective dispersion coefficient. 
TABLE I. Variation of different dimensionless parameters and the corresponding physical parameter that is varied.

\begin{tabular}{lcl}
\hline \hline Fixed & Varying & \multicolumn{1}{c}{ Physical parameter } \\
\hline$\ell / H, \ell / \delta$ & $\mathrm{Pe}$ & Flow speed $\left(u_{0}\right)$ \\
$\ell / H, \ell / \delta=\infty\left(D_{T}=0\right)$ & $\mathrm{Pe}_{\dot{\gamma}}$ & Flow speed \\
$\ell / H, \mathrm{Pe}_{\dot{\gamma}}$ & $\ell / \delta$ & Translational diffusivity $\left(D_{T}\right)$ \\
$\beta, D_{T}=0$ & $\mathrm{Pe}_{\dot{\gamma}}$ & Reorientation time $\left(\tau_{R}\right)$ \\
$\mathrm{Pe}_{\dot{\gamma}}, \mathrm{Pe}=\infty\left(D_{T}=0\right)$ & $\beta$ & Swim speed $\left(U_{0}\right)$ \\
$\mathrm{Pe}_{\dot{\gamma}}, \mathrm{Pe}$ & $\beta$ & Swim speed \\
$\beta, \mathrm{Pe}$ & $\mathrm{Pe}$ & Translational diffusivity \\
$\beta, \mathrm{Pe}$ & $\mathrm{Pe}_{\dot{\gamma}}$ & Reorientation time \\
\hline
\end{tabular}

Substituting the small wave-number expansion into Eq. (13), we obtain at leading order

$$
\frac{\partial g_{0}}{\partial t}+\frac{\partial}{\partial y}\left(U_{0} q_{y} g_{0}-D_{T} \frac{\partial g_{0}}{\partial y}\right)-D_{R} \nabla_{R}^{2} g_{0}+\nabla_{R} \cdot\left(\frac{1}{2} \omega g_{0}\right)=0 .
$$

At order $i k$, we obtain the governing equation for the fluctuating field $d_{0}$ :

$$
\frac{\partial d_{0}}{\partial t}+\frac{\partial}{\partial y}\left(U_{0} q_{y} d_{0}-D_{T} \frac{\partial d_{0}}{\partial y}\right)-D_{R} \nabla_{R}^{2} d_{0}+\nabla_{R} \cdot\left(\frac{1}{2} \omega d_{0}\right)=\left(U^{\mathrm{eff}}-u-U_{0} q_{x}\right) g_{0} .
$$

The no-flux boundary conditions for $g_{0}$ and $d_{0}$ at $y= \pm H / 2$ are given by

$$
U_{0} q_{y} g_{0}-D_{T} \frac{\partial g_{0}}{\partial y}=0 \quad \text { and } \quad U_{0} q_{y} d_{0}-D_{T} \frac{\partial d_{0}}{\partial y}=0
$$

The normalization condition translates into

$$
\frac{1}{H} \int d y \int g_{0} d \mathbf{q}=1 \quad \text { and } \quad \frac{1}{H} \int d y \int d_{0} d \mathbf{q}=0 .
$$

At this point, the continuum formulation is complete. The governing equations are characterized by three dimensionless groups: the strength of confinement $\ell / H=U_{0} \tau_{R} / H$, the activity level $\ell / \delta=$ $U_{0} \tau_{R} / \sqrt{D_{T} \tau_{R}}$, and the Péclet number Pe $=u_{0} H / D_{T}$. The Péclet number characterizes the relative importance of advection by flow and translational diffusion. To quantify the effect of flow speed $u_{0}$ on the drift and dispersion, one can vary Pe while keeping $\ell / H$ and $\ell / \delta$ fixed. However, it is cumbersome to characterize the effect of $U_{0}, \tau_{R}$, or $D_{T}$ separately because all of them appear in two dimensionless parameters. We therefore introduce two alternate dimensionless groups:

$$
\beta=\frac{U_{0}}{u_{0}} \quad \text { and } \quad \mathrm{Pe}_{\dot{\gamma}}=\overline{\dot{\gamma}} \tau_{R}=\frac{2 u_{0} \tau_{R}}{H},
$$

where $\overline{\dot{\gamma}}=\overline{|d u / d y|}=2 u_{0} / H$ is the average shear rate across the channel. The first parameter $\beta$ is a direct comparison of the swim speed of the ABPs and the flow speed at the centerline of the channel. The second parameter $\mathrm{Pe}_{\dot{\gamma}}$ is a Péclet number that compares the two time scales of reorientation: rotational Brownian motion on the scale of $\tau_{R}$ and rotation from fluid shear with a time scale $1 / \bar{\gamma}$. Using $\beta, \mathrm{Pe}$, and $\mathrm{Pe}_{\dot{\gamma}}$ as independent dimensionless parameters, we can conveniently probe the effect of $U_{0}, D_{T}$, and $\tau_{R}$ separately. For example, variation of the swim speed $U_{0}$ is fully characterized by variation of $\beta$ while both $\mathrm{Pe}$ and $\mathrm{Pe}_{\dot{\gamma}}$ are fixed. Similarly, variation of $\tau_{R}$ is represented by variation of $\mathrm{Pe}_{\dot{\gamma}}$ while $\beta$ and Pe are fixed. To measure the effect of $D_{T}$, we can vary $\mathrm{Pe}$ and fix both $\mathrm{Pe}_{\dot{\gamma}}$ and $\beta$. The two dimensionless parameters $\ell / H$ and $\ell / \delta$ can be expressed in terms of $\beta, \mathrm{Pe}$, and $\mathrm{Pe}_{\dot{\gamma}}$ via the relations $\ell / H=\beta \mathrm{Pe}_{\dot{\gamma}} / 2, \ell / \delta=\beta \sqrt{\mathrm{PePe}_{\dot{\gamma}} / 2}$. Conversely, we have $\beta=(\ell / \delta)^{2}(\ell / H)^{-1} \mathrm{Pe}^{-1}$ and $\mathrm{Pe}_{\dot{\gamma}}=2 \mathrm{Pe}(\ell / H)^{2}(\ell / \delta)^{-2}$. In Table I, we summarize the different 
schemes of varying dimensionless groups and their corresponding physical situation in terms of dimensional parameters. In experiments, some variation schemes are easier than others. For example, one could easily vary the flow speed and quantify the transport of active particles as a function of Pe for a given $\ell / \delta$ and $\ell / H$.

Henceforth, we consider ABPs in two dimensions where the orientation vector $\mathbf{q}$ is in the velocity-gradient plane. As a result, one can parametrize $\mathbf{q}$ in terms of a single orientation angle $\theta \in[0,2 \pi)$ where $\mathbf{q}=\cos \theta \mathbf{e}_{x}+\sin \theta \mathbf{e}_{y}$. We note that the qualitative behavior of the average drift and longitudinal dispersion does not depend on the dimensionality of the orientation space. We shall discuss three separate methods to solve (15)-(21): truncated orientational moment expansion, solution of (18) and (19) using the finite element method (FEM), and BD simulation. For the first two methods, we determine $g_{0}$ from (18) and the average drift $U^{\text {eff }}$ from (15). With solution to $g_{0}$ and $U^{\text {eff }}$, we then determine $d_{0}$ from (19) and $D^{\text {eff }}$ from (16).

As a first approach, we approximate the average and fluctuating field in terms of their truncated orientational moments [52,53]:

$$
g_{0} \approx \frac{1}{2 \pi}\left(n^{0}+2 \mathbf{q} \cdot \mathbf{m}^{0}+4 \mathbf{q q}: \mathbf{Q}^{0}\right), \quad d_{0} \approx \frac{1}{2 \pi}(\tilde{n}+2 \mathbf{q} \cdot \tilde{\mathbf{m}}+4 \mathbf{q q}: \tilde{\mathbf{Q}}),
$$

where $\mathbf{Q}^{0}=\int(\mathbf{q q}-\mathbf{I} / 2) g_{0} d \mathbf{q}$ is the average nematic field and $\tilde{\mathbf{Q}}=\int(\mathbf{q q}-\mathbf{I} / 2) d_{0} d \mathbf{q}$ is the fluctuating nematic field. Substituting these expressions for $g_{0}$ and $d_{0}$ and taking orientational moments of appropriate order yields a set of closed ordinary differential equations at steady state for the average and fluctuating number density and polar and nematic fields (refer to Appendix A for details). Mathematically, this approximation can be interpreted as a severely truncated Fourier series expansion of $g_{0}$ and $d_{0}$ in the orientation angle. The moment expansion converges rapidly only when the probability distributions are near isotropy.

For the problem of this paper, the distribution can be very anisotropic in the presence of a Poiseuille flow. It is then beneficial to solve the governing equations for $g_{0}$ and $d_{0}$ directly and then take the needed orientational moments to obtain the average drift and effective dispersion coefficient. To this end, we solve (18) and (19) directly with a finite element method implemented using FREEFEM++ [54] with adaptive mesh refinement. In the FEM formulation, the periodic boundary condition in $\theta$ is enforced, $g_{0}(y, \theta=0, t)=g_{0}(y, \theta=2 \pi, t)$, and $d_{0}(y, \theta=0, t)=$ $d_{0}(y, \theta=2 \pi, t)$. After the system reaches steady state, the orientational moments of $g_{0}$ and $d_{0}$ are calculated according to (17) and then the drift and effective dispersion coefficient are obtained using (15) and (16).

From a particle-level perspective, the evolution of the configuration of ABPs can be described by the overdamped Langevin equations. For each $\mathrm{ABP}$, this force and torque balance is given by

$$
\begin{gathered}
\mathbf{0}=-\zeta\left(\frac{d \mathbf{x}}{d t}-\mathbf{u}\right)+\mathbf{F}^{B}+\mathbf{F}^{S}, \\
\mathbf{0}=-\zeta_{R}\left(\frac{d \mathbf{q}}{d t}-\frac{1}{2} \boldsymbol{\omega} \times \mathbf{q}\right)+\mathbf{L}^{B} \times \mathbf{q} .
\end{gathered}
$$

Here, $\mathbf{F}^{S}=\zeta U_{0} \mathbf{q}$ is the swim force [17], and $\mathbf{F}^{B}\left(\mathbf{L}^{B}\right)$ is the Brownian force (torque). $\zeta$ and $\zeta_{R}$ are the translational and rotational hydrodynamic drag coefficients, respectively. The Brownian force and torque satisfy white-noise statistics:

$$
\begin{aligned}
\left\langle\mathbf{F}^{B}\right\rangle=0, & \left\langle\mathbf{F}^{B}(0) \mathbf{F}^{B}(t)\right\rangle=2 D_{T} \zeta^{2} \delta(t) \mathbf{I}, \\
\left\langle\mathbf{L}^{B}\right\rangle=0, & \left\langle\mathbf{L}^{B}(0) \mathbf{L}^{B}(t)\right\rangle=\frac{2}{\tau_{R}} \zeta_{R}^{2} \delta(t) \mathbf{I},
\end{aligned}
$$

where $\delta(t)$ is the delta function and the angle brackets denote the ensemble average over Brownian fluctuations. We emphasize that the translational and rotational diffusion coefficients represent biological noises and their variation can be independent. 
We evolve the Langevin equation with a typical Brownian dynamics algorithm [5] for approximately $10^{5}$ noninteracting particles to ensure good statistics. The duration of the BD simulation was chosen to be longer than the slowest time scale in the problem so that the long-time behavior is captured. The domain of simulation is a square box of side length $H$ with periodic boundary condition in the flow direction and hard walls at $y= \pm H / 2$. The hard-wall boundary condition is implemented using the potential-free algorithm [55] that ensures that the particle does not cross the wall. The interaction of the particle with the wall does not change the particle orientation. The absolute position of each ABP is recorded to calculate the effective drift and diffusivity. Further details of the BD simulation are presented in Appendix B.

\section{UPSTREAM SWIMMING}

The drift $U^{\text {eff }}$ quantifies the average motion of ABPs along the channel due to the combined effects of advection by the ambient flow and self-propulsion. In the absence of flow $\left(u_{0} \equiv 0\right)$, the notion of upstream or downstream is lost and the longitudinal polarization $m_{x}^{0}$ vanishes by symmetry. As a result, the drift $U^{\text {eff }}$ is zero if the flow is not present. The introduction of an ambient flow breaks the left-right symmetry in the longitudinal direction, which enables nonzero polarization $\left(m_{x}^{0} \neq 0\right)$. This symmetry breaking ultimately leads to the tendency of ABPs to swim upstream in a pressure-driven flow, which has been investigated experimentally $[29,31,32]$ and theoretically [36]. A number of mechanisms for the upstream swimming of micro-organisms have been proposed including chirality of flagellar bundles and hydrodynamic interactions [32,56-59]. However, even for the simplest case of spherical particles without fluid-mediated or particle-particle interactions as we consider here, upstream swimming is still present. In the following section, by considering the case of non-Brownian particles, we show that this peculiar upstream swimming behavior can be explained with simple physical arguments: A self-propelling particle reaching the wall points into the wall. The body-fixed swimming director $\mathbf{q}$ is then rotated towards the upstream direction owing to the strong fluid vorticity at the wall. As a result, the particle moves upstream.

Though the moment equations are not analytically tractable, a number of symmetry properties can be obtained. First, solution to the Smoluchowski equation (18) along with the boundary condition (20) satisfies the symmetry property that $g_{0}(y, \theta, t)=g_{0}(-y, 2 \pi-\theta, t)$. Integrating over the orientation, we have $n^{0}(y)=n^{0}(-y)$, i.e., the number density is an even function of $y$. Similarly, one can show that $m_{y}^{0}(-y)=-m_{y}^{0}(y)$ (and therefore, $\overline{m_{y}^{0}} \equiv 0$ ) and $m_{x}^{0}(-y)=m_{x}^{0}(y)$. Taking the cross-sectional average of the steady-state moment equation (A3), we can further obtain $\overline{m_{x}^{0}}=-\frac{1}{2} \tau_{R} \overline{\omega m_{y}^{0}}$. This expression shows that the vorticity induces a coupling between the polar order in the flow direction and that in the transverse direction.

\section{A. Non-Brownian active particles}

We first consider non-Brownian particles with $D_{T}=0$ and $D_{R}=0$ (or $\tau_{R}=\infty$ ). In this case, the active particle has an infinite run length $\ell=U_{0} \tau_{R}=\infty$. Without translational and rotational Brownian motion, an active particle follows a deterministic trajectory. Zöttl and Stark [34] examined the nonlinear dynamics of non-Brownian microswimmers in Poiseuille flow in a cylindrical tube. Both upstream and downstream trajectories are observed depending on the flow speed. When the flow speed is small, the microswimmer that comes into contact with the wall is turned upstream and performs a swinging motion between the walls while swimming upstream. Here, we examine this interesting behavior in more detail and quantify the transition between upstream and downstream motion in the absence of Brownian motion. The equations of motion given by Eqs. (24) and (25) reduce to $d x / d t=u+U_{0} \cos \theta, d y / d t=U_{0} \sin \theta$, and $d \theta / d t=\frac{1}{2} \omega$, where $\omega=8 u_{0} y / H^{2}$ is the scalar vorticity. The unit orientation vector is written in a parametric form, $\mathbf{q}=\cos \theta \mathbf{e}_{x}+\sin \theta \mathbf{e}_{y}$. We note that a second-order equation for the orientation angle can be obtained: $d^{2} \theta / d t^{2}=$ $4 u_{0} U_{0} \sin \theta / H^{2}$. In general, the particle trajectory depends on its initial position and orientation. If the particle is located on the centerline $\left(y_{0}=0\right)$ and aligned with the flow $\left(\theta_{0}=0\right)$ initially, 

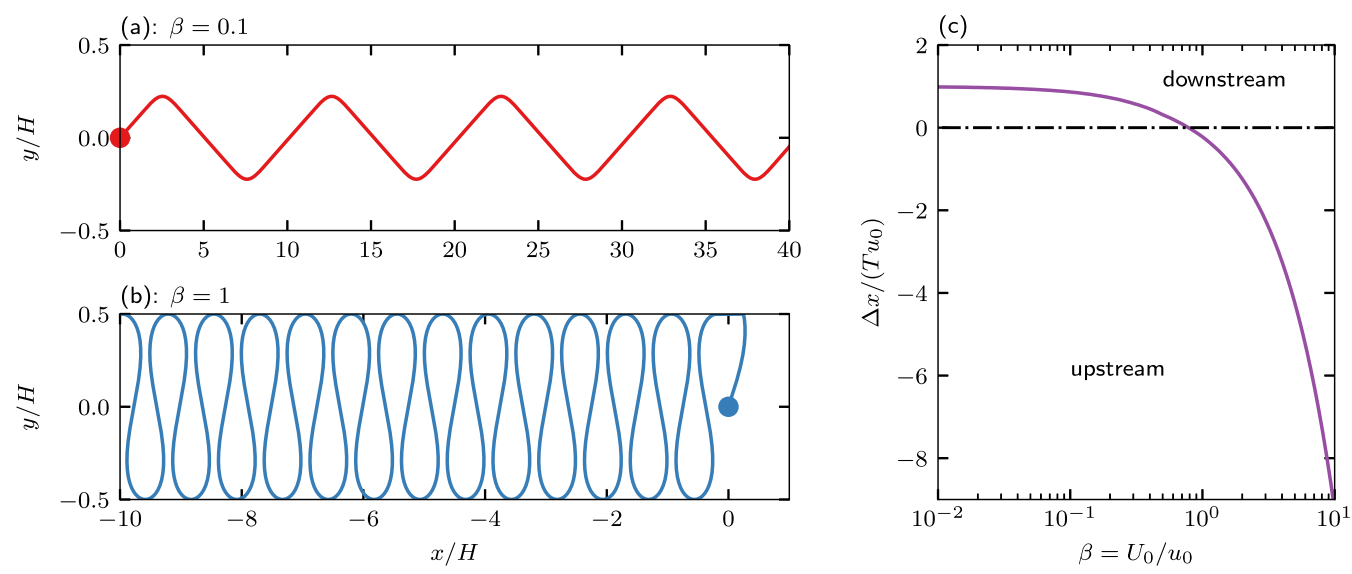

FIG. 5. (a), (b) Trajectories of a non-Brownian active particle for different speed ratios $\beta=U_{0} / u_{0}$. The initial positions are marked by dots. (c) Average speed in the longitudinal direction as a function of $\beta$. Initial conditions for all three panels are identical: $x_{0}=0, y_{0}=0, \theta_{0}=\pi / 2$.

the equations of motion can be solved exactly to obtain $\theta \equiv 0, y \equiv 0$ and $x(t)=x_{0}+\left(u_{0}+U_{0}\right) t$. Because the torque due to shear vanishes on the centerline, the particle will not rotate, and thus stays on the centerline for all time. Starting from this initial condition, the particle will always move downstream. On the other hand, if the particle is located on the centerline but aligned against the flow $(\theta=\pi)$ at $t=0$, the solution becomes $x(t)=x_{0}+\left(u_{0}-U_{0}\right) t$. Depending on the relative magnitude of self-propulsion and fluid advection $\left(\beta=U_{0} / u_{0}\right)$, the particle can move upstream $(\beta>1)$ or downstream $(\beta<1)$. The upstream-downstream transition occurs at $\beta=1$, where the particle will be stationary for all time. We note that the upstream motion of a non-Brownian active particle with initial condition $y_{0}=0$ and $\theta_{0}=\pi$ is a stable fixed point while the downstream motion with $y_{0}=0$ and $\theta_{0}=0$ is an unstable fixed point. In general, the swimming behavior is different from this special case and the fluid vorticity plays a major role in the orientation dynamics of the active particle.

For arbitrary initial conditions, we solve the equations of motion numerically using the explicit Runge-Kutta method (RK4). Interaction of the particle with the channel wall is treated using the potential-free algorithm [55] as discussed earlier. We reiterate that the interaction of the particle with the wall specified by the potential-free algorithm does not change the particle orientation. If the swim speed is small compared to the flow speed $(\beta \ll 1)$, the active particle will always move downstream. In the small $\beta$ limit, due to its slow swimming speed, the active particle cannot reach the wall before its direction changes significantly due to the fluid shear. A typical particle trajectory in this advection-dominated regime is presented in Fig. 5(a).

For large $\beta$, the active particle located on the channel centerline can travel to the wall if it is not aligned in the longitudinal direction. Due to the large fluid vorticity at the wall, the particle will be reorientated upstream. The continuing reorientation and swimming result in periodic movement between the walls and net upstream motion. A particle trajectory in this regime is shown in Fig. 5(b). Similar trajectories to Figs. 5(a) and 5(b) are found in Zöttl and Stark [34]. To quantify the net motion of a particle, we present the net speed in the longitudinal direction scaled by the flow speed as a function of $\beta$ in Fig. 5(c). The net speed is given by $\Delta x / T$, where $\Delta x$ is the distance traveled in the longitudinal direction within a period $(T)$ of its motion. The period $T$ is obtained using autocorrelation of the orientation angle $\theta$. As expected, the particle moves downstream at small $\beta$ and upstream at large $\beta$.

It is instructive to examine this upstream-swimming trajectory in more detail. In Fig. 6(a) we show the $y$ coordinate and orientation angle $\theta$ of the active particle as a function of the scaled time 

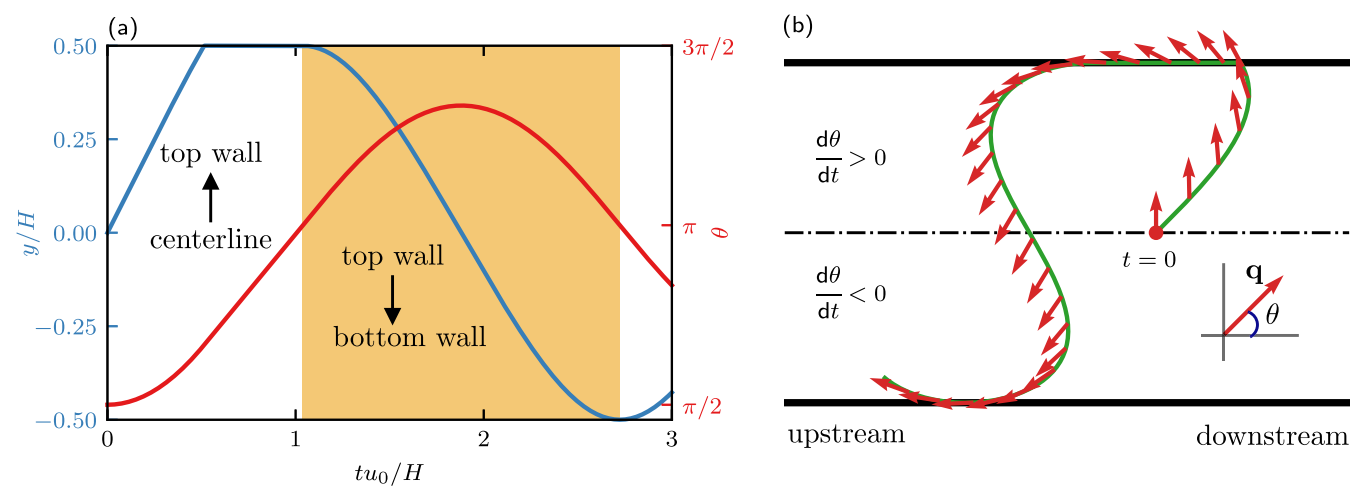

FIG. 6. (a) Initial trajectory $y / H$ (blue, left axis) and orientation angle $\theta$ (red, right axis) as a function of the dimensionless time $t u_{0} / H$ for a non-Brownian active particle. The shaded region represents the time period in which the particle moves from the top wall to the bottom wall. (b) Initial trajectory (solid, green line) and orientation vector (red, arrows) of the active particle in the channel. Particles rotate counterclockwise $(d \theta / d t>0)$ in the upper half of the channel and clockwise $(d \theta / d t<0)$ in the lower half of the channel. Parameters: $y_{0}=0, \theta_{0}=\pi / 2$, and $\beta=1$.

$t u_{0} / H$. To better visualize the swimming behavior, the same particle trajectory in the $x-y$ plane (green line) with red arrows denoting the orientation vector is shown in Fig. 6(b). Initially, the particle is located on the centerline and pointed towards the top wall. As time starts, the particle moves to the top wall while being rotated counterclockwise by the fluid vorticity and advected downstream. If the swim speed $U_{0}$ is large enough compared to $u_{0}$, the particle is able to arrive at the top wall where the fluid vorticity is the greatest. Because the particle cannot penetrate through the hard wall, it remains at the wall until the vorticity rotates it towards the upstream so that it can finally escape into the bulk. As the particle escapes into the bulk, it now points towards the bottom wall and starts to swim towards it. Once the particle reaches the lower half of the channel, the fluid vorticity reverses sign and the particle is again rotated towards the upstream direction. It will then approach the bottom wall if its speed $U_{0}$ permits. Once the particle reaches the bottom wall, the above process repeats itself and we observe a periodic upstream trajectory confined between the two walls at later times.

In this section, we considered the transport of a self-propelled non-Brownian particle in a pressure driven flow. Specifically, we studied the swimming behavior of non-Brownian active particles that start on the centerline. We note that a particle starting from a location other than the centerline exhibits similar behavior to those shown in Fig. 6. With a sufficiently large speed of self-propulsion, an active particle is able to swim upstream rather than being advected downstream by the flow. The interplay of biased rotation due to the fluid vorticity and confinement gives rise to this interesting upstream swimming behavior.

\section{B. No translational diffusion}

We now consider the case in which the ABPs have no translational diffusion $\left(D_{T}=0\right)$ but finite rotational diffusion $\left(D_{R} \neq 0\right)$. In the Smoluchowski perspective, the absence of translational diffusion is singular. A diffusive flux at the wall is required to balance the flux due to self-propulsion. In the limit of vanishing $D_{T}$, a boundary layer develops at the walls [28]. Starting from the Smoluchowski equation, one can reformulate the problem in a singular perturbation approach and obtain equations inside the boundary layer and out in the bulk. In the presence of flow, the resulting equations are not analytically tractable. Instead, we make use of the BD simulation introduced earlier to probe this regime of zero translational diffusion. 

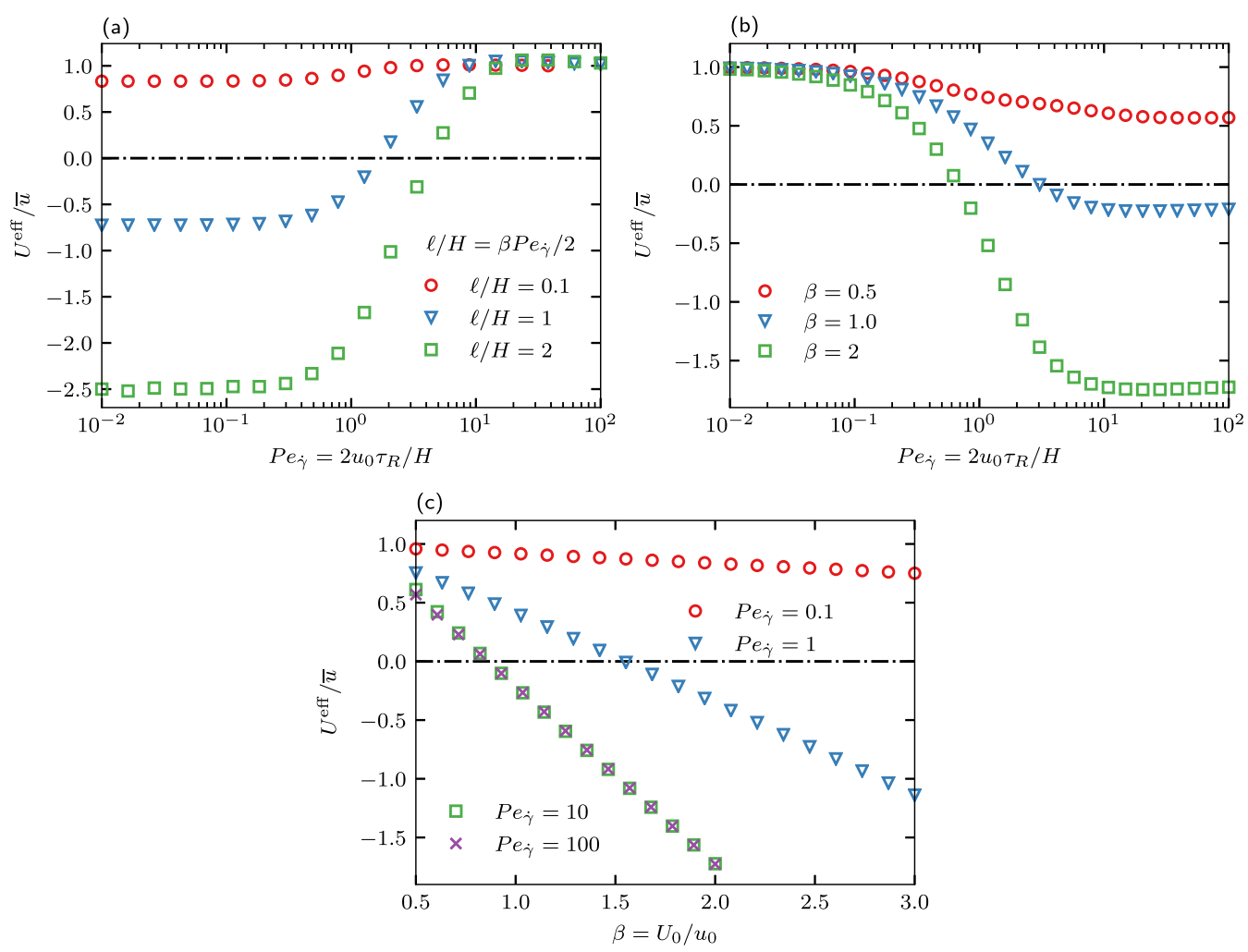

FIG. 7. Variation of the average drift $U^{\mathrm{eff}} / \bar{u}$ in the absence of translational diffusion $\left(D_{T}=0\right)$ as a function of (a) the flow speed $u_{0}$, (b) the reorientation time $\tau_{R}$, and (c) the swim speed $U_{0}$. (a) Drift $U^{\text {eff }} / \bar{u}$ as a function of $\mathrm{Pe}_{\dot{\gamma}}=2 u_{0} \tau_{R} / H$ for $\ell / H=\{0.1,1,2\}$. (b) Drift $U^{\text {eff }} / \bar{u}$ as a function of $\mathrm{Pe}_{\dot{\gamma}}$ for $\beta=U_{0} / u_{0}=\{0.5,1,2\}$. (c) Drift $U^{\text {eff }} / \bar{u}$ as a function of $\beta$ for $\mathrm{Pe}_{\dot{\gamma}}=\{0.1,1,10,100\}$. The horizontal dash-dotted lines denote the transition between downstream and upstream drift.

For $D_{T}=0$, we have $\ell / \delta=\infty$ and $\mathrm{Pe}=\infty$ given that all other dimensional parameters are nonzero and finite. In this case, $\beta=U_{0} / u_{0}$ and $\mathrm{Pe}_{\dot{\gamma}}=2 u_{0} \tau_{R} / H$ are used as the two independent dimensionless groups. Recall the relation $\ell / H=\beta \mathrm{Pe}_{\dot{\gamma}} / 2$. In Fig. 7(a), we show the average drift $U^{\text {eff }} / \bar{u}$ as a function of $\mathrm{Pe}_{\dot{\gamma}}$ for different values of the confinement strength $\ell / H$. For a fixed $\ell / H$, the variation of $\mathrm{Pe}_{\dot{\gamma}}$ is better interpreted as a variation of the flow speed at the centerline $u_{0}$ while other dimensional parameters are fixed. If the flow speed $u_{0} \equiv 0$, the average drift $U^{\text {eff }}$ vanishes by symmetry. We note that, however, $U^{\text {eff }} / \overline{u_{0}}$ does not vanish in the limit $u_{0} \rightarrow 0$ as shown in Fig. 7(a). When the flow is weak $\left(\mathrm{Pe}_{\dot{\gamma}} \ll 1\right)$, active particles accumulate and swim upstream at the wall, which makes the average drift less than the average flow speed $\left(U^{\text {eff }}<\bar{u}\right)$. We emphasize that the causes for this reduction of drift are twofold: first, the number density is higher at the wall than at the centerline, which implies that $\overline{u n^{0}}<\bar{u}$ because the flow speed is zero at the wall and is the greatest at the centerline; second, a negative polar order in the flow direction develops, which means that $U_{0} \overline{m_{x}^{0}}<0$. For small $\ell / H$, the swimming speed is not strong enough to overcome the downstream advection, i.e., $\left|U_{0} m_{x}^{0}\right|<\overline{u n^{0}}$, and we obtain a downstream drift. For larger $\ell / H$, upstream swimming is dominant and an upstream drift is achieved as shown in Fig. 7(a) for $\ell / H=$ $\{1,2\}$.

For $\mathrm{Pe}_{\dot{\gamma}} \gg 1$ with $\ell / H$ fixed, the flow speed $u_{0}$ is much faster than the swim speed $U_{0}$. From (15), we have $U^{\text {eff }} / \bar{u}=3 \beta \overline{m_{x}^{0}} / 2+\overline{u n^{0}} / \bar{u} \rightarrow \overline{u n^{0}} / \bar{u}$ since $\beta \rightarrow 0$ as $\mathrm{Pe}_{\dot{\gamma}} \rightarrow \infty$. Furthermore, particles in 
this strong flow regime spin rapidly owing to the dominant fluid vorticity compared to Brownian reorientation. This leads to a more uniform number density distribution across the channel and thus $U^{\text {eff }} / \bar{u}$ approaches unity in the large $\mathrm{Pe}_{\dot{\gamma}}$ limit.

In Fig. 7(b), we show the average drift $U^{\text {eff }} / \bar{u}$ as a function of $\mathrm{Pe}_{\dot{\gamma}}$ for different values of the speed ratio $\beta$ in the absence of translational diffusion. For a fixed $\beta$, this represents the effect of the reorientation time $\tau_{R}$ on the drift. For $\mathrm{Pe}_{\dot{\gamma}} \ll 1$, the reorientation time $\tau_{R}$ is much smaller than the fluid shear timescale $1 / \bar{\gamma}$, and particles lose their persistence due to the rapid random reorientation and behave like passive particles without self-propulsion. As a result, the effective velocity approaches the passive limit $U^{\text {eff }} / \bar{u} \rightarrow 1$ as $\mathrm{Pe}_{\dot{\gamma}} \rightarrow 0$ regardless of the speed ratio $\beta$.

For $\mathrm{Pe}_{\dot{\gamma}} \gg 1$, the reorientation time $\tau_{R}$ is large and particles follow nearly deterministic trajectories with small fluctuations from Brownian rotation. One can recover the purely ballistic case discussed in the previous section by taking the limit $\mathrm{Pe}_{\dot{\gamma}} \rightarrow \infty$ with a finite $\beta$ (e.g., $\tau_{R} \rightarrow \infty$ ).

It is important to distinguish between Brownian reorientation and rotation by fluid vorticity. Brownian reorientation is random and unbiased while the rotation due to the fluid vorticity is deterministic and biased. In order to move upstream, active particles have to be aligned against the flow so that they can swim upstream due to self-propulsion. This orientational bias towards the upstream, as discussed in the previous section, is achieved by the deterministic rotation from the fluid vorticity in the presence of a confining boundary. With other parameters fixed, Brownian reorientation becomes weaker as $\tau_{R}$ increases $\left(D_{R}\right.$ decreases, $\mathrm{Pe}_{\dot{\gamma}}$ increases), and therefore is unable to randomize the biased orientation generated by the fluid vorticity. As a result, the average drift shown in Fig. 7(b) decreases monotonically as a function of $\mathrm{Pe}_{\dot{\gamma}}$, becoming negative-upstream swimming — and asymptotes to the non-Brownian limit at large $\mathrm{Pe}_{j}$.

We showed the variation of $U^{\mathrm{eff}} / \bar{u}$ as a function of $\mathrm{Pe}_{\dot{\gamma}}$ with different dimensionless parameters being fixed in Figs. 7(a) and 7(b). When $\ell / H$ is fixed, upstream drift is observed for small $\mathrm{Pe}_{\gamma}$. On the other hand, upstream drift is achieved for large $\mathrm{Pe}_{\dot{\gamma}}$ when $\beta$ is fixed. We emphasize that to interpret the results properly one has to be precise about what parameters are fixed.

In addition to the two competing reorientation mechanisms characterized by $\mathrm{Pe}_{\dot{\gamma}}$, the speed ratio $\beta$ also plays an important role in determining the swimming behavior. In Fig. 7(c), we show the variation of $U^{\text {eff }} / \bar{u}$ as a function of $\beta$ for different values of $\mathrm{Pe}_{\dot{\gamma}}$. For small $\beta$ (e.g., $\beta=0.5$ ), active particles move downstream $\left(U^{\text {eff }} / \bar{u}>0\right)$ for all values of $\mathrm{Pe}_{\dot{\gamma}}$. The transition from downstream to upstream swimming $\left(U^{\text {eff }} / \bar{u}<0\right)$ is observed for larger $\beta$ only. For a given $\mathrm{Pe}_{\dot{\gamma}}$, the average drift $U^{\text {eff }} / \bar{u}$ decreases monotonically and becomes negative as $\beta$ increases. We note that for $\beta=$ 0 , i.e., the particles are passive, the orientational degree of freedom becomes decoupled from the translational, and we recover the passive limit: $U^{\text {eff }} / \bar{u} \rightarrow 1$ as $\beta \rightarrow 0$.

\section{Finite translational and rotational diffusion}

We now examine the distribution and swimming behavior of ABPs with finite translational and rotational diffusivities in the presence of a pressure-driven flow. To illustrate the effect of translational diffusivity $\left(D_{T}\right)$ on the behavior of active particles, we show in Fig. 8 the average number density $n^{0}$ (recall that $n^{0}=\int g_{0} d \mathbf{q}$ ), average streamwise polar order $m_{x}^{0}$, and average probability distribution $g_{0}(y=H / 2, \theta)$ for different $\mathrm{Pe}=u_{0} H / D_{T}$ with $\beta=U_{0} / u_{0}=2$ and $\mathrm{Pe}_{\dot{\gamma}}=$ $2 u_{0} \tau_{R} / H=10$. The $D_{T}=0$ case discussed in the previous section is the limit $\mathrm{Pe} \rightarrow \infty$ and $\beta, \mathrm{Pe}_{j}<\infty$. With $\beta$ and $\mathrm{Pe}_{\dot{\gamma}}$ fixed at finite values, the variation of translational diffusivity $D_{T}$ is represented in dimensionless form by the variation of Pe. In other words, Pe goes down as $D_{T}$ increases and $\mathrm{Pe} \ll 1$ implies that the translational diffusion dominates over the fluid advection. In this strong diffusion limit, the steady-state probability profile cannot sustain a large gradient across the channel due to the smoothing effect of diffusion. As a result, in Figs. 8(a) and 8(b) we observe a mostly uniform number density and polar order for $\mathrm{Pe}=0.1$. As Pe increases, translational diffusion becomes less important and we observe the development of boundary layers at the wall in both the average number density and polar order distribution. At large Pe, ABPs exhibit strong accumulation and upstream polarization at the wall. In Fig. 8(c), the average field $g_{0}$ at the top wall is plotted 
(a)



(b)

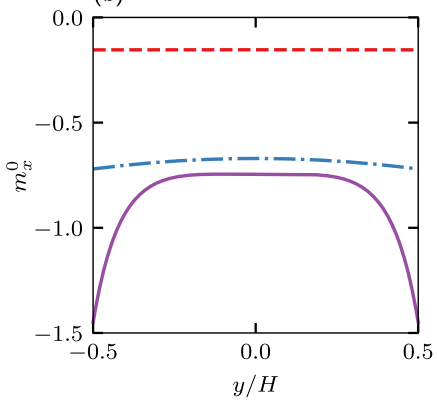

(c)

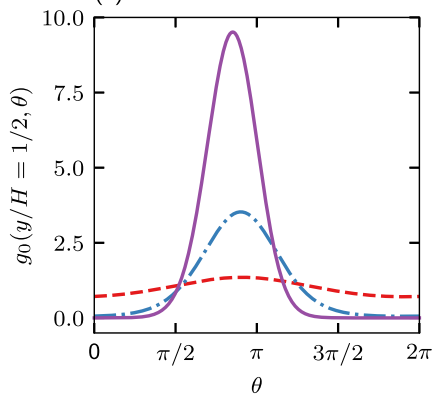

FIG. 8. Effect of translational diffusivity $D_{T}$ on the average field distributions. (a) Average number density distribution across the channel for different values of $\mathrm{Pe}=u_{0} H / D_{T}$. (b) Average streamwise polar order distribution across the channel for different values of Pe. (c) The average field $g_{0}$ as a function of $\theta$ at the top wall $(y / H=1 / 2)$ for different values of Pe. The solutions presented are obtained from the finite element calculation with $\beta=U_{0} / u_{0}=2$ and $\mathrm{Pe}_{\dot{\gamma}}=2 u_{0} \tau_{R} / H=10$.

as a function of the orientation angle $\theta$ for different Péclet numbers. Recall that an ABP with the orientation angle $\theta=\pi / 2$ is pointing normally into the top wall and an ABP with $\theta=\pi$ is pointing upstream parallel to the wall. In the strong diffusion limit $(\mathrm{Pe} \ll 1)$, only a weak variation of $g_{0}$ as a function of $\theta$ is observed. Similar to the orientational order distribution across the channel, the probability at the wall develops a large gradient and the peak shifts towards the upstream direction as Pe increases.

We note that even though the streamwise polar order $m_{x}^{0}<0$ for $\mathrm{Pe}=0.1$ it is not strong enough to overcome the fluid advection, which leads to a net downstream motion $\left(U^{\text {eff }}>0\right)$. The dependence of the average drift $U^{\text {eff }} / \bar{u}$ on Pe is made quantitative in Fig. 9. Here, the dashed lines are solutions from the moment equations, the circles are solutions from FEM and the crosses are solutions from BD simulations. The horizontal dash-dotted line represents the drift velocity of passive particles, $U^{\text {eff }}=\bar{u}$. Results from BD and FEM agree very well while solution from the moment equations deviates from BD (or FEM) for $\mathrm{Pe}_{\dot{\gamma}}=10$ at large Pe as seen in Fig. 9(a). The


FIG. 9. (a) Average drift $U^{\text {eff }} / \bar{u}$ of ABPs as a function of $\mathrm{Pe}=u_{0} H / D_{T}$ for $\mathrm{Pe}_{\dot{\gamma}}=2 u_{0} \tau_{R} / H=1$ (red) and $\mathrm{Pe}_{\dot{\gamma}}=10$ (blue) with fixed $\beta=U_{0} / u_{0}=2$. (b) Average drift $U^{\text {eff }} / \bar{u}$ of ABPs as a function of the Péclet number for $\ell / H=U_{0} \tau_{R} / H=1$ (red) and $\ell / H=10$ (blue) with fixed $\ell / \delta=U_{0} \tau_{R} / \sqrt{D_{T} \tau_{R}}=30$. Circles are from FEM, while dashed lines denote results from the moment equations. Results from Brownian dynamics are indicated by crosses. The dash-dotted green line is the effective drift of passive particles. 
moment method truncating after the nematic order is not sufficient to capture this behavior. One can show that our simple truncated expansions of the average and fluctuation fields given in Eq. (23) lead to isotropic closures to the third moments of $g_{0}$ and $d_{0}$ [52]. Because this truncation does not incorporate the effect of the external flow field even though particles rotate according to Jeffery's equation, it is only a good approximation when the average and fluctuation fields are not far away from isotropy. Therefore, care must be taken when using these isotropic closures in the presence of an external flow.

For fixed finite $\beta$ and $\mathrm{Pe}_{\dot{\gamma}}$ as shown in Fig. 9(a), the increase of Pe is understood as the decrease in the translational diffusivity $D_{T}$. The effective drift $U^{\text {eff }} / \bar{u}$ approaches unity in the limit $\mathrm{Pe} \rightarrow 0$ $\left(D_{T} \rightarrow \infty\right)$ because the particle number density becomes uniform owing to strong translational diffusion. For small Pe, ABPs have a net downstream motion. A transition from downstream to upstream motion occurs at a larger Pe where more particles accumulate at the wall. Because the fluid vorticity is the greatest at the wall while the fluid velocity is zero, particles at the wall are able to swim upstream with a larger net speed. As a result, for a given $\beta$ and $\mathrm{Pe}_{\dot{\gamma}}$, the effective drift velocity $U^{\text {eff }} / \bar{u}$ decreases monotonically as a function of Pe and reaches a plateau at large Pe. The limit of $\mathrm{Pe} \rightarrow \infty$ with fixed $\beta, \mathrm{Pe}_{\dot{\gamma}}<\infty$ asymptotes to the case of no translational diffusion explored in the previous section.

The variation of $U^{\mathrm{eff}} / \bar{u}$ as a function of Pe depends qualitatively on how the parameter space is sampled. By keeping $\beta$ and $\mathrm{Pe}_{\dot{\gamma}}$ constant as shown in Fig. 9(a), the variation of Pe is understood as the variation of the translational diffusivity alone. In Fig. 9(b), to investigate the effect of the flow speed $u_{0}$ on the average drift, we fix $\ell / \delta=U_{0} \tau_{R} / \sqrt{D_{T} \tau_{R}}$ and $\ell / H=U_{0} \tau_{R} / H$ and vary Pe. In this case, Pe increases as the flow speed $u_{0}$ increases. Noting that $\ell / \delta$ characterizes the activity level of ABPs, a suspension with $\ell / \delta=30$ is in the highly active regime. The competition between upstream swimming and downstream advection gives rise to a nonmonotonic variation of the drift velocity as a function of Péclet number. As discussed earlier, the effective drift vanishes $\left(U^{\text {eff }}=0\right)$ when the flow is absent $(P e=0)$. For weak flow $(P e \ll 1)$, upstream swimming dominates over downstream advection and the drift becomes negative. Upstream swimming is particularly strong for large confinement, e.g., $\ell / H=10$ in Fig. 9(b). In the strong flow (Pe $\gg 1$ ) limit, the advection of ABPs by the ambient flow is much stronger than the self-propelling speed. Besides, the accumulation of $\mathrm{ABPs}$ at the wall is greatly reduced as Pe increases. As a result, the drift velocity approaches that of a passive particle for large Pe. As a function of the flow speed, the average drift $U^{\text {eff }}$ of ABPs with high activity first decreases to a negative value and then increases to approach the passive limit $\bar{u}$. We note that the ratio $U^{\text {eff }} / \bar{u}$ approaches a nonzero value in the limit $u_{0} \rightarrow 0$. Because we scaled the dimensional average drift using $\bar{u}$, the ratio $U^{\text {eff }} / \bar{u}$ is a monotonically increasing function of Pe as shown in Fig. 9(b). We note that the nonmonotonicity of the average drift refers to the variation of the dimensional quantity $U^{\text {eff }}$ as a function of Pe. In Appendix C, the effect of nonspherical shape on the average drift of ABPs is discussed.

In Fig. 10, we present the average polar order distribution in the $x$ direction $\left(m_{x}^{0}\right)$ across the channel for different Péclet numbers for a very active suspension $(\ell / \delta=30)$ with $\ell / H=10$. As the flow strength $(\mathrm{Pe})$ increases, upstream swimming becomes weaker. For $\mathrm{Pe}=10$, the time scale of rotation by flow vorticity at the wall and random rotation by Brownian motion are comparable $\left[\mathrm{Pe}_{j}=2 \mathrm{Pe}(\ell / H)^{2}(\ell / \delta)^{-2} \approx 2.22\right]$. In this regime, the flow vorticity at the wall only biases the orientation of ABPs towards the upstream. In the strong flow limit $(\mathrm{Pe} \gg 1)$, the time scale for rotation by flow vorticity is much smaller than the Brownian reorientation time $\tau_{R}$ even in the bulk. As a result, the persisting motion of an ABP vanishes because of the fast spinning due to vorticity.

This fast spinning by vorticity also affects the number density distribution. At large Pe where flow is strong, ABPs at the wall are quickly reoriented by vorticity without relying on rotary diffusion to escape into the bulk flow. This leads to a more uniform distribution of particles across the channel at large Pe. In Fig. 11, we present the average number density distribution $n^{0}$ across the channel for different Péclet numbers with $\ell / H=10$ and $\ell / \delta=30$. Indeed, we observe a reduction of wall accumulation as the Péclet number increases. The reduction of wall accumulation in the presence of flow has also been observed by Chilukuri et al. [60] for a model microswimmer consisting of 

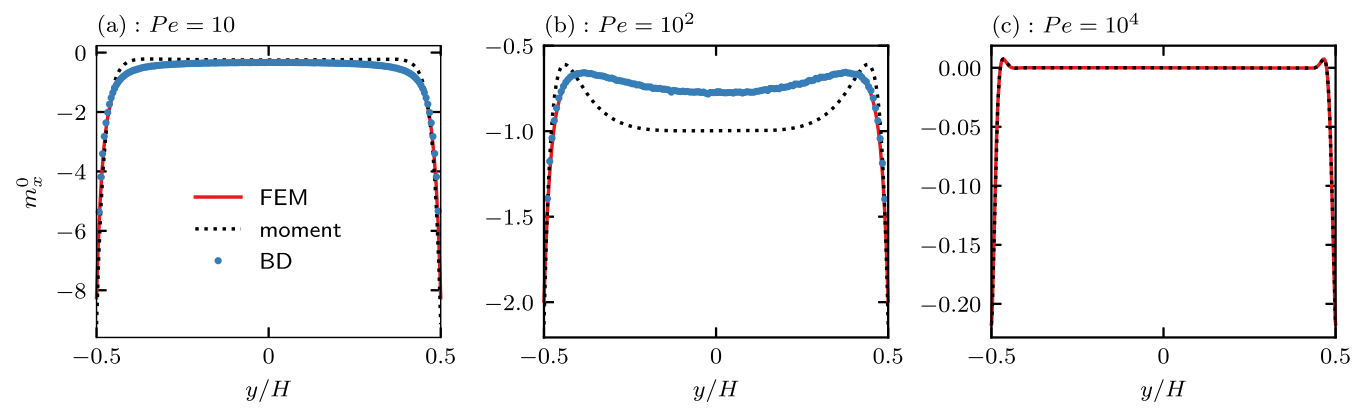

FIG. 10. The average polar order distribution $\left(m_{x}^{0}\right)$ across the channel for different Péclet numbers $(\mathrm{Pe}=$ $u_{0} H / D_{T}$ ) with $\ell / H=U_{0} \tau_{R} / H=10$ and $\ell / \delta=U_{0} \tau_{R} / \sqrt{D_{T} \tau_{R}}=30$. The red solid lines are solutions from FEM, the black dashed lines are from moment equations, and the blue dots are results from BD simulation.

two beads connected by a stiff spring. Another interesting feature is the nonmonotonic variation of number density across the channel at large Péclet number. At $\mathrm{Pe}=100$, a weak accumulation of particles at the channel centerline is observed. This centerline accumulation is due to strong confinement. If we decrease $\ell / H$ or increase $\ell / \delta$, the bulk number density will become more uniform. For $\mathrm{Pe}=10^{4}$, Fig. 11(c), a local depletion of particles is observed near the wall before the number density increases at the wall. In bulk flow, the number density is uniform due to the vanishing swimming motion. In Brownian dynamics simulation, we observe that ABPs can be trapped near the wall when the flow is strong. An ABP approaching the upper wall will be rotating counterclockwise. Instead of escaping into the bulk once orientated upstream, an ABP comes back to the wall again due to the rapid rotation by flow vorticity. It will repeat this process until random Brownian motion kicks it outside this region and it can escape to the bulk. As a result, we still observe a small amount of wall accumulation and a region of depletion because ABPs here either go back to the wall or escape into the bulk.

\section{LONGITUDINAL DISPERSION}

We now turn our attention to the effect of flow on longitudinal dispersion of ABPs in a channel. The average drift considered in the previous section characterizes the mean motion of ABPs along the channel. In contrast, the effective dispersivity measures the variation of particle motion in the longitudinal direction compared to the mean. In this section, we first discuss the effective
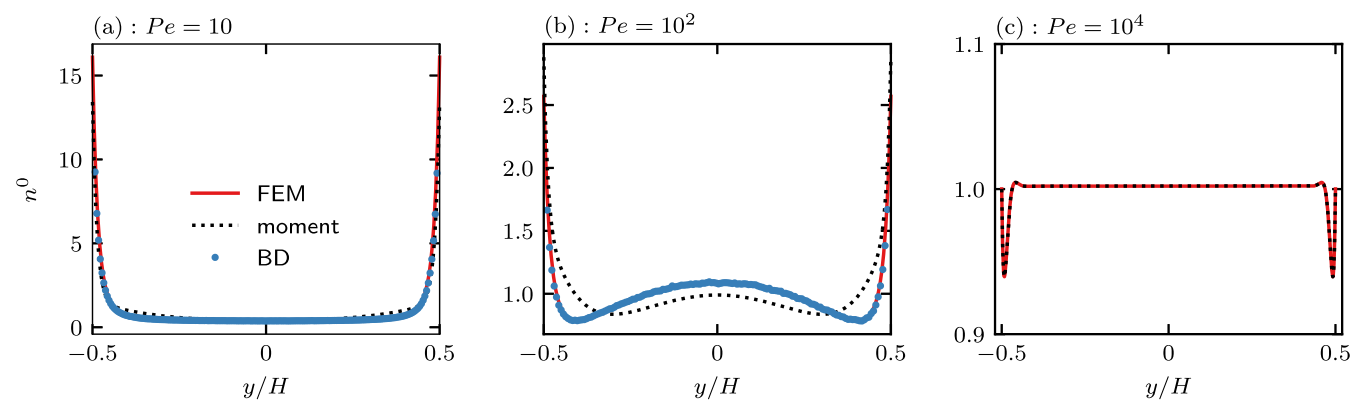

FIG. 11. The average number density distribution $\left(n^{0}\right)$ across the channel for different Péclet numbers $\left(\mathrm{Pe}=u_{0} H / D_{T}\right)$ with $\ell / H=U_{0} \tau_{R} / H=10$ and $\ell / \delta=U_{0} \tau_{R} / \sqrt{D_{T} \tau_{R}}=30$. The red solid lines are solutions from FEM, the black dashed lines are from moment equations, and the blue dots are results from BD simulation. 
longitudinal dispersion when the translational diffusivity $D_{T}=0$, in which case the classical Taylor dispersion is absent. We then discuss the general case with finite $D_{T}$.

\section{A. Dispersion in the absence of translational diffusion}

In this section, we explore the long-time longitudinal diffusive behavior of active particles in the presence of flow in the absence of translational diffusion, $D_{T}=0$. For passive particles with $D_{T}=0$, there is no long-time diffusive motion. Their behavior is purely deterministic and ballistic, that is, being advected downstream with the local flow speed. But for active particles, there is longtime diffusive behavior from the coupling between the diffusive sampling of orientation space due to Brownian rotation and the orientational dependence of the self-propelling velocity $U_{0} \mathbf{q}$. In other words, the dispersion of active particles with $D_{T}=0$ consists of the swim diffusivity $D^{\text {swim }}$ alone while the presence of an external flow modulates the effective speed in a run and the effective reorientation time $\tau_{\text {eff }}$ of the effective long-time random-walk process. Recall that the longitudinal dispersion coefficient becomes the free space swim diffusivity $\left(D^{\text {eff }}=D_{0}^{\text {swim }} \equiv U_{0}^{2} \tau_{R} / 2\right)$ if the flow is also absent $\left(u_{0}=0\right)$. This coupling of rotation to translation does not exist for passive particles that do not self-propel. Therefore, Brownian sampling of the orientation space becomes irrelevant to the consideration of the effective dispersion for passive particles.

To reveal the effect of flow on the longitudinal dispersion of active particles, we show in Fig. 12(a) the effective dispersion coefficient scaled by the free space swim diffusivity as a function of $\mathrm{Pe}_{\dot{\gamma}}=2 u_{0} \tau_{R} / H$ for different strengths of confinement $\ell / H=U_{0} \tau_{R} / H$. The same quantity is plotted as a function of $\mathrm{Pe}_{\dot{\gamma}}$ for different values of $\beta=U_{0} / u_{0}$ in Fig. 12(b) and as a function of $\beta$ for different values of $\mathrm{Pe}_{\dot{\gamma}}$ in Fig. 12(c). Dash-dotted horizontal lines represent the case in which the effective dispersivity is identical to that in free space, $D^{\text {eff }}=D_{0}^{\text {swim }}$. For fixed $\ell / H$ as shown in Fig. 12(a), the variation of $\mathrm{Pe}_{\dot{\gamma}}$ is understood as the variation of the flow speed $u_{0}$. For fixed $\beta$ as shown in Fig. 12(b), the variation of $\mathrm{Pe}_{\dot{\gamma}}$ is the variation of the reorientation time $\tau_{R}$. In Fig. 12(c) where $\mathrm{Pe}_{j}$ is fixed, the variation of $\beta$ corresponds to the variation of the swim speed $U_{0}$.

In the presence of a pressure-driven flow, the effective longitudinal dispersivity is altered in an interesting and nontrivial fashion. As shown in Fig. 12(a), the effective dispersivity $D^{\text {eff }} / D_{0}^{\text {swim }}$ is a nonmonotonic function of the flow speed: the effective dispersivity $D^{\text {eff }}$ can be either enhanced $\left(D^{\text {eff }} / D_{0}^{\text {swim }}>1\right)$ or hindered $\left(D^{\text {eff }} / D_{0}^{\text {swim }}<1\right)$ compared to $D_{0}^{\text {swim }}$. To understand this nonmonotonic behavior, consider the effective long-time random-walk process of the ABPs in the presence of pressure-driven flow. In addition to the fluid vorticity that modifies the effective reorientation time $\tau_{\text {eff }}$, the fluid advection affects the effective speed in a run. Recall that the effect of vorticity on orientation is characterized by $\mathrm{Pe}_{j}$ and the effect of flow speed is characterized by $\beta$. For a given $\ell / H, \beta$ decreases as the flow speed (i.e., $\mathrm{Pe}_{\dot{\gamma}}$ ) increases since $\ell / H=\beta \mathrm{Pe}_{\dot{\gamma}} / 2$. When the flow is weak $\left(\mathrm{Pe}_{\gamma} \ll 1\right)$, we have $\beta \gg 1$ and the advection is dominated by swimming and we recover the free space swim diffusivity, $D^{\text {eff }} \rightarrow U_{0}^{2} \tau_{R} / 2$ as $\mathrm{Pe}_{\dot{\gamma}} \rightarrow 0$ with $\ell / H$ fixed. For strong flow $\left(\mathrm{Pe}_{\dot{\gamma}} \gg 1\right)$, the effective reorientation time is reduced owing to the fluid vorticity but the effective speed in a run increases due to fluid advection. The effect of fluid advection dominates and the longitudinal dispersion is greatly enhanced.

The two competing effects originating from the background flow give rise to the nonmonotonic behavior. For $\ell / H=0.1$, the fluid advection becomes important $[\beta \sim O(1)]$ for even small $\mathrm{Pe}_{\dot{\gamma}}$ $(\sim 0.1)$ where the effect of the fluid vorticity is still weak. In this case, the dispersivity increases monotonically as a function of $\mathrm{Pe}_{j}$. For $\ell / H=\{1,2\}$, the effect of vorticity becomes important at $\mathrm{Pe}_{\dot{\gamma}} \sim O(1)$ and we observe an initial decrease in the dispersivity due to a reduction of the effective reorientation time. As the fluid advection becomes dominant $(\beta<1), \mathrm{Pe}_{\dot{\gamma}} \sim 10$, the dispersivity increases as $\mathrm{Pe}_{\dot{\gamma}}$ increases.

In the large $\mathrm{Pe}_{\dot{\gamma}}$ limit for $\ell / H=0.1$, we observe a strong dependence of the effective longitudinal dispersion on the flow speed, $D^{\text {eff }} / D_{0}^{\text {swim }} \sim \mathrm{Pe}_{\dot{\gamma}}^{4}$. Interestingly, this giant longitudinal dispersion has also been observed by Dehkharghani et al. [48] in the dispersion of active particles with $D_{T}=0$ in flow through a periodic porous media. Due to the rapid spinning from the fluid vorticity, active 

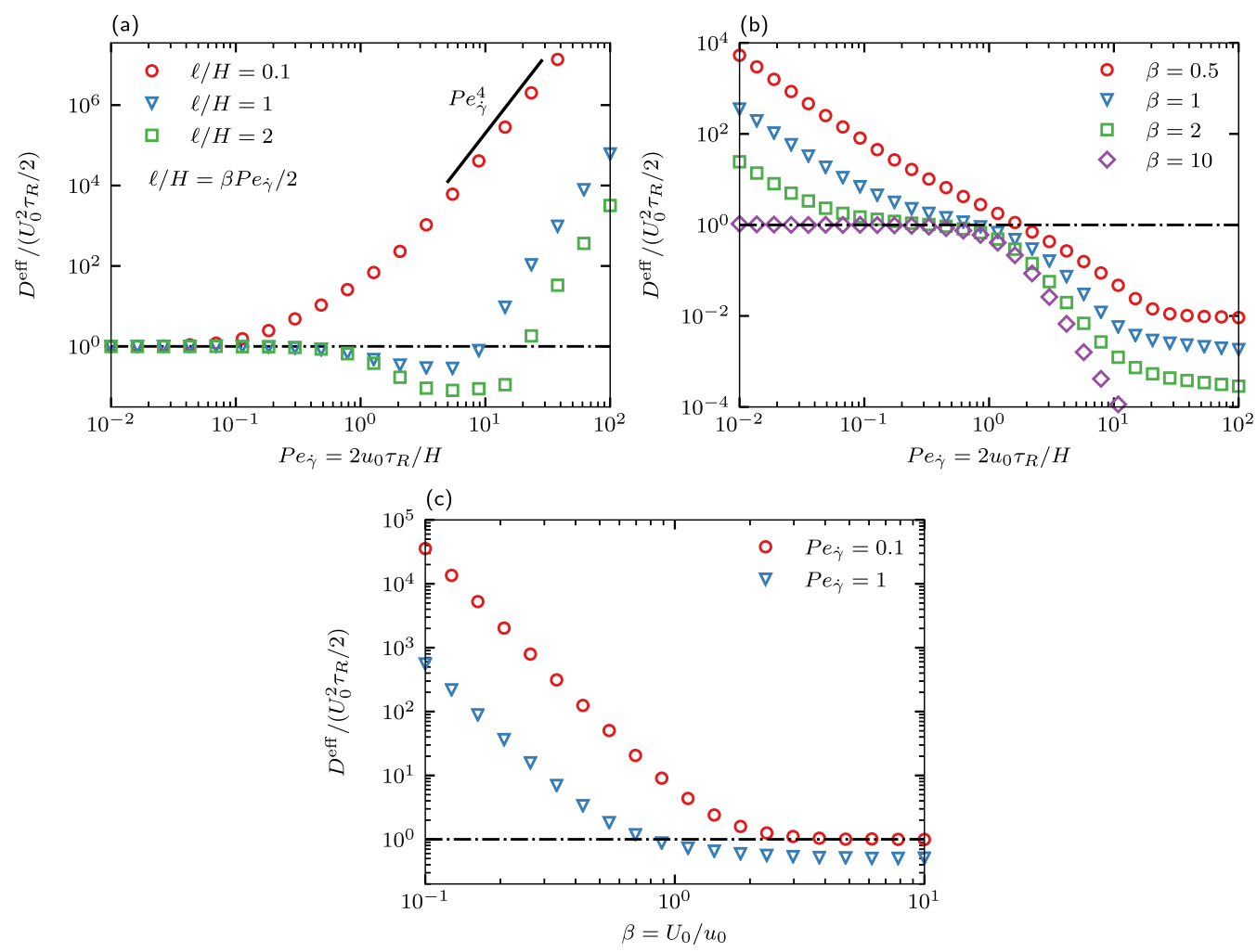

FIG. 12. Variation of the effective longitudinal dispersivity $D^{\text {eff }} /\left(U_{0}^{2} \tau_{R} / 2\right)$ in the absence of translational diffusion $\left(D_{T}=0\right)$ as a function of (a) the flow speed $u_{0}$, (b) the reorientation time $\tau_{R}$, and (c) the swim speed $U_{0}$. (a) Effective longitudinal dispersivity $D^{\text {eff }} /\left(U_{0}^{2} \tau_{R} / 2\right)$ as a function of $\mathrm{Pe}_{\dot{\gamma}}=2 u_{0} \tau_{R} / H$ for $\ell / H=U_{0} \tau_{R} / H=\{0.1,1,2\}$. (b) Effective longitudinal dispersivity $D^{\text {eff }} /\left(U_{0}^{2} \tau_{R} / 2\right)$ as a function of $\mathrm{Pe}_{\dot{\gamma}}$ for $\beta=U_{0} / u_{0}=\{0.5,1,2,10\}$. (c) Effective longitudinal dispersion coefficient $D^{\text {eff }} /\left(U_{0}^{2} \tau_{R} / 2\right)$ as a function of $\beta$ for $\mathrm{Pe}_{\dot{\gamma}}=\{0.1,1\}$. The results shown are obtained from BD simulations. The horizontal dash-dotted lines are $D^{\text {eff }}=U_{0}^{2} \tau_{R} / 2$, i.e., the free space swim diffusivity.

particles are not confined in the transverse direction since their effective run length $\ell^{\text {eff }}$ is reduced such that $\ell^{\text {eff }} \ll H$. In a constant vorticity field in an unbounded domain, the transverse dispersion coefficient of active particles is reduced. In the large $\mathrm{Pe}_{\dot{\gamma}}$ limit, this reduction follows the scaling $D_{\perp} / D_{0}^{\text {swim }} \sim 1 / \mathrm{Pe}_{\dot{\gamma}}^{2}[18,48]$. Making use of the scaling in the classical Taylor dispersion process, $D^{\text {eff }} \sim u_{0}^{2} H^{2} / D_{\perp}$, we have $D^{\text {eff }} / D_{0}^{\text {swim }} \sim u_{0}^{2} H^{2} \mathrm{Pe}_{\dot{\gamma}}^{2} /\left(U_{0}^{4} \tau_{R}^{2}\right) \sim \mathrm{Pe}_{\dot{\gamma}}^{4}(\ell / H)^{-4}$ in the large $\mathrm{Pe}_{\dot{\gamma}}$ limit. For a fixed $\ell / H$, we recover the scaling $D^{\text {eff }} / D_{0}^{\text {swim }} \sim \mathrm{Pe}_{\dot{\gamma}}^{4}$. In Fig. 12 (a) for $\ell / H=0.1$, this limiting behavior is achieved for $\mathrm{Pe}_{\dot{\gamma}}>10$. For larger $\ell / H$, we expect the same quartic scaling in $\mathrm{Pe}_{\dot{\gamma}}$ as $\mathrm{Pe}_{\dot{\gamma}} \rightarrow \infty$. In the range of $\mathrm{Pe}_{\dot{\gamma}}$ sampled in Fig. 12(a), this scaling is not achieved yet for $\ell / H=1,2$.

To explore the effect of the reorientation time $\tau_{R}$, in Fig. 12(b) we fix $\beta$ and plot the longitudinal dispersivity as a function of $\mathrm{Pe}_{\dot{\gamma}}$. Physically, the variation of $\mathrm{Pe}_{\dot{\gamma}}$ with $\beta$ fixed corresponds to the variation of the importance of the fluid vorticity while keeping the advective effect of the flow fixed. For $\mathrm{Pe}_{\dot{\gamma}}<1$, Brownian reorientation is the fast time scale and rotation by the fluid vorticity is less important. On the other hand, rotation by the fluid vorticity becomes dominant for $\mathrm{Pe}_{\dot{\gamma}}>1$. The effective reorientation time $\tau_{\text {eff }}$ is reduced and thus the dispersivity decreases monotonically as $\mathrm{Pe}_{\dot{\gamma}}$ increases. For $\mathrm{Pe}_{\dot{\gamma}} \ll 1$, the dominant reorientation mechanism is Brownian and the enhancement of longitudinal dispersion for small $\beta$ is due to the increase in the effective speed in the random walk from the fluid advection. The no-flow limit where $D^{\text {eff }} \rightarrow D_{0}^{\text {swim }}$ is recovered as the flow speed 
(a): $\ell / \delta=2$, low activity

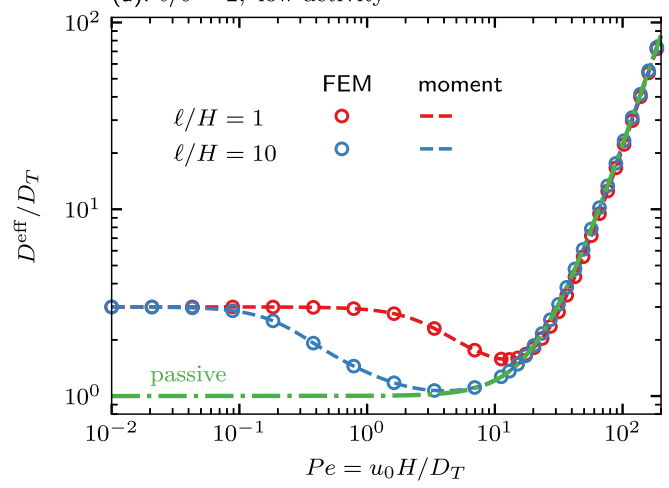

(b): $\ell / \delta=30$, high activity

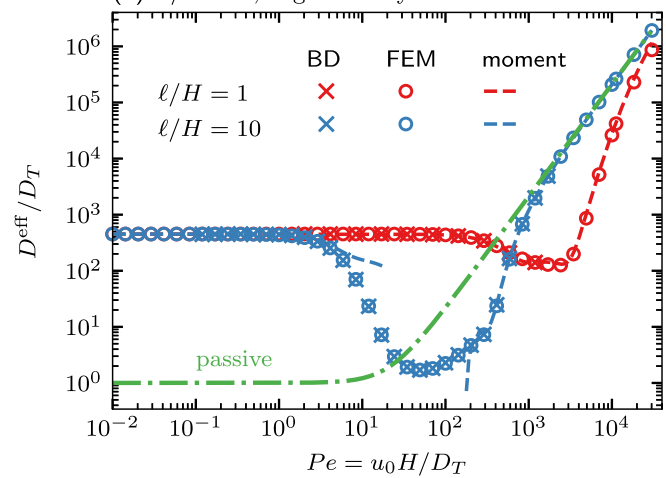

FIG. 13. Effective longitudinal dispersivity $D^{\text {eff }} / D_{T}$ as a function of $\mathrm{Pe}=u_{0} H / D_{T}$ for (a) $\ell / \delta=$ $U_{0} \tau_{R} / \sqrt{D_{T} \tau_{R}}=2$ and (b) $\ell / \delta=30$ and different confinement strengths (red, $\ell / H=U_{0} \tau_{R} / H=1$; blue, $\ell / H=10$ ). Circles are results from FEM and dashed lines are solutions from moment equations. Results from BD simulation are marked by cross symbols. In regions of comparably large activity and flow strength, the moment equations do not agree with FEM or BD simulation. The classical dispersion for passive particles is plotted in a dash-dotted green line.

$u_{0} \rightarrow 0$, which in terms of the dimensionless parameters is obtained by taking the limit $\beta \rightarrow \infty$ and $\mathrm{Pe}_{\dot{\gamma}} \rightarrow 0$. This no-flow limit is effectively recovered with $\beta=10$ and $\mathrm{Pe}_{\dot{\gamma}} \ll 1$ as marked by the diamond symbols in Fig. 12(b).

At this point the difference between Taylor dispersion of passive matter and active particles without translational diffusion should be noted. In the passive case, the longitudinal dispersion is always enhanced by the flow. In contrast, the pressure-driven flow can either enhance or hinder the longitudinal dispersion of active particles.

In Fig. 12(c), we show the dispersivity as a function of the speed ratio $\beta$ for fixed values of $\mathrm{Pe}_{\dot{\gamma}}$. This corresponds to a variation of the effect of the fluid advection while keeping the effect of the fluid vorticity fixed. In terms of dimensional parameters, this is a variation of the swim speed $U_{0}$ while keeping other parameters fixed. As the swim speed increases, the effective run speed transitions from the fluid speed to the swim speed and the effective longitudinal dispersion decreases monotonically. The limiting value of the dispersivity in the large $\beta$ limit depends on $\mathrm{Pe}_{\dot{\gamma}}$. If $\mathrm{Pe}_{\dot{\gamma}}$ is small (e.g., $\left.\mathrm{Pe}_{\dot{\gamma}}=0.1\right)$, Brownian reorientation dominates and we recover the no-flow limit $\left(D_{0}^{\text {swim }}\right)$. If $\mathrm{Pe}_{\dot{\gamma}}$ is larger (e.g., $\mathrm{Pe}_{\dot{\gamma}}=1$ ), the effective reorientation time is reduced and the longitudinal dispersivity is less than $D_{0}^{\text {swim }}$.

\section{B. Dispersion with finite translational diffusion}

We now consider the general case of ABPs with finite translational diffusion. For active particles with $D_{T}=0$ as explored in the previous section, the background flow affects longitudinal particle dispersion through the rotation by vorticity in orientation space and advection in physical space. In addition to this effective modulation of the swim diffusivity, ABPs with finite $D_{T}$ also experience the classical Taylor dispersion that occurs in physical space alone. Therefore, the dispersion of ABPs reflects the combined effects of the pressure-driven flow on the particle dynamics in both orientational and physical space.

For given activity level $\ell / \delta$ and confinement strength $\ell / H$, the variation of Pe corresponds to the variation of the flow speed $u_{0}$ with other dimensional quantities being fixed. We present in Fig. 13(a) the effective longitudinal dispersivity $D^{\text {eff }} / D_{T}$ as a function of Péclet number for a suspension with low activity, $\ell / \delta=2$, and two different confinement strengths $\ell / H=\{1,10\}$. The same quantities are presented in Fig. 13(b) for a very active suspension characterized by $\ell / \delta=30$. 
Similar to the variation of the effective drift $U^{\text {eff }} / \bar{u}$ as a function of Pe with fixed $\ell / \delta$ and $\ell / H$ shown in Fig. 9(b), we observe a nonmonotonic variation of the effective longitudinal dispersivity $D^{\text {eff }} / D_{T}$ as a function of the Péclet number. Notice that the effective dispersivity has three contributions, the translational diffusivity $\left(D_{T}\right)$, fluctuation of the streamwise swimming motion $\left(-U_{0} \overline{\tilde{m}_{x}}\right)$, and fluctuation in the number density weighted by the flow $(-\overline{u \tilde{n}})$. Recall that in the absence of $D_{T}$ only the fluctuation in the swimming motion remains and the effective dispersivity reduces to the swim diffusivity if the flow speed is also zero. For small Péclet number, the flow is very weak and the dispersion is dominated by the fluctuation in self-propulsion. We have $D^{\text {eff }} \rightarrow D_{T}+D_{0}^{\text {swim }}$ as $\mathrm{Pe} \rightarrow 0$. The dispersivity in this region is insensitive to the variation in confinement $\ell / H$ as discussed earlier for the no-flow case $(\mathrm{Pe}=0)$.

In the large Péclet number limit, the advective effect dominates and the effective dispersion approaches that of the classical Taylor dispersion of passive particles. A minimum in the effective dispersivity is obtained when the effect of advection by the ambient flow is comparable to the self-propulsion of ABPs.

To understand the physical origin of this nonmonotonic variation in the effective dispersivity, we consider two separate problems. First, by neglecting the advection $(\mathbf{u}=\mathbf{0})$, we remove the effect of classical Taylor dispersion and consider the effect of vorticity alone. As one increases strength of the vorticity, the effective reorientation time decreases due to the spinning motion while the random-walk speed is unchanged. This reduced effective reorientation time gives rise to decreasing swim diffusivity. As $\mathrm{Pe} \rightarrow \infty$ for fixed finite $\ell / \delta$ and $\ell / H, \mathrm{Pe}_{\dot{\gamma}} \rightarrow \infty$, and the swim diffusivity $D^{\text {swim }} \rightarrow 0$. Next, we neglect the swimming motion of ABPs $\left(U_{0}=0\right)$ and simply recover the classical Taylor dispersion problem. In the classical Taylor dispersion problem, the effective dispersivity increases monotonically as a function of the Péclet number. By combining these two separate problems, the total effective dispersivity becomes nonmonotonic as a function of the Péclet number. In other words, increasing of the flow strength diminishes the swim diffusivity while at the same time generates shear-induced dispersion. It is this competition that gives rise to the nonmonotonic variation in the effective longitudinal dispersion of ABPs in a pressure-driven flow.

The above physical argument suggests the consideration of $\mathrm{Pe}_{\dot{\gamma}}$ that characterizes how strong the biased rotation by vorticity is compared to random Brownian reorientation. Noting that $\mathrm{Pe}_{\dot{\gamma}}$ is proportional to $\mathrm{Pe}$ for fixed $\ell / H$ and $\ell / \delta$ from the relation $\operatorname{Pe}_{\dot{\gamma}}=2 \operatorname{Pe}(\ell / H)^{2}(\ell / \delta)^{-2}$. We can define a critical condition $\mathrm{Pe}_{\dot{\gamma}} \sim 1$ where the rotational effect of the fluid vorticity becomes comparable to Brownian reorientation. If we consider a fixed activity level [e.g., $\ell / \delta=30$ shown in Fig. 13(b)], we can predict the relative location of the minimal effective dispersivity when the confinement $\ell / H$ varies. For a fixed $\ell / \delta$, we require $(\ell / H)_{1}^{2} \mathrm{Pe}_{1} \sim(\ell / H)_{2}^{2} \mathrm{Pe}_{2}$ where the subscripts 1 and 2 denote different confinement strengths. Using parameters in Fig. 13(b), we have $\mathrm{Pe}_{2} / \mathrm{Pe}_{1} \sim 10^{2}$. Here, subscript 1 denotes $\ell / H=10$ and 2 denotes $\ell / H=1$. Similarly, we can consider a fixed confinement, say $\ell / H=1$, and different activity level. By the same argument, we have $\mathrm{Pe}_{2} / \mathrm{Pe}_{1} \sim$ 900 with 2 denoting $\ell / \delta=30$ and 1 being $\ell / \delta=1$.

To recover the classical Taylor dispersion analytically, we set $U_{0}=0$ in the moment equations and obtain $n^{0} \equiv 1$. In other words, the average number density of passive particles is uniform across the channel as expected. The average drift is simply the average flow velocity $U^{\text {eff }}=2 u_{0} / 3$. The fluctuating field number density can then be obtained:

$$
\tilde{n}(y)=-\frac{u_{0} H^{2}}{720 D_{T}}\left[7-120\left(\frac{y}{H}\right)^{2}+240\left(\frac{y}{H}\right)^{4}\right]
$$

Taking an average of $\tilde{n}$ weighted by the flow field, we recover the effective longitudinal dispersivity for passive particles $D^{\text {eff }} / D_{T}=1+2 P e^{2} / 945$. The numerical factor is different from that given in Ref. [15] due to their definition of the Péclet number based on the average instead of the maximum flow velocity. The effective dispersivity for passive particles as a function of Péclet number is plotted 


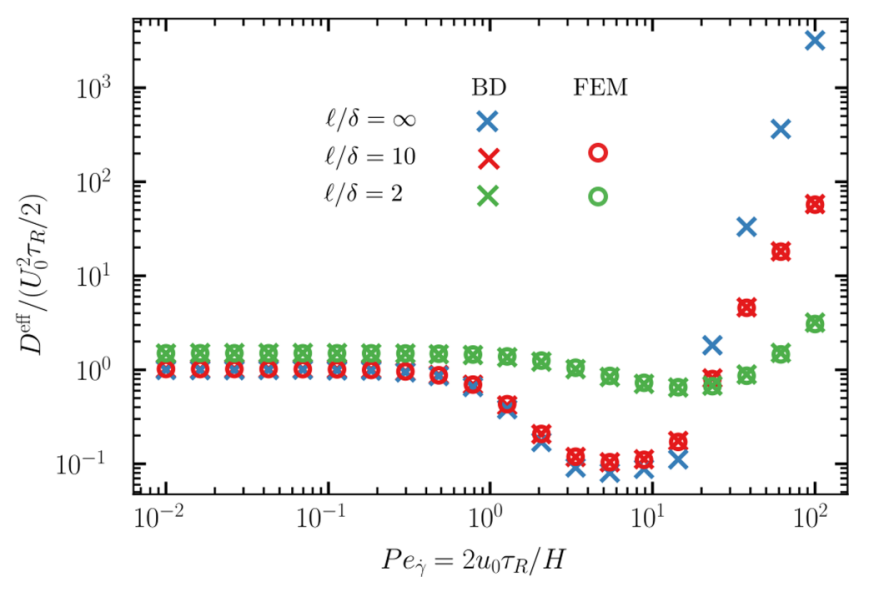

FIG. 14. The effective longitudinal dispersion coefficient $D^{\text {eff }} /\left(U_{0}^{2} \tau_{R} / 2\right)$ as a function of $\mathrm{Pe}_{\dot{\gamma}}$ for different values of $\ell / \delta$. The degree of confinement is fixed, $\ell / H=2$. For a given $\mathrm{Pe}_{\dot{\gamma}}$ and $\ell / H$, decreasing $\ell / \delta$ corresponds to increasing the translational diffusivity.

in Fig. 13 with a dash-dotted green line. In Appendix C, the effect of nonspherical shape on the longitudinal dispersion of ABPs is considered.

In Fig. 14, we show the variation of $D^{\text {eff }} / D_{0}^{\text {swim }}$ as a function of $\mathrm{Pe}_{\dot{\gamma}}$ for $\ell / H=2$ and different values of $\ell / \delta$. The blue cross markers are results for active particles with $D_{T}=0(\ell / \delta=\infty)$, which has been shown in Fig. 12(a) with square markers. Decreasing $\ell / \delta$ corresponds to increasing the translational diffusivity of the ABPs. The strong nonmonotonic variation of the effective dispersion coefficient as a function of the flow speed $\left(\mathrm{Pe}_{\dot{\gamma}}\right)$ is observed when translational diffusion is weak $\left(\ell / \delta\right.$ is large). Due to the presence of classical Taylor dispersion for active particles with finite $D_{T}$, the nonmonotonicity becomes weaker as $D_{T}$ increases $(\ell / \delta$ decreases).

\section{CONCLUSION}

In this paper, we have presented a combined analytical and numerical investigation of the effective longitudinal advection and dispersion of noninteracting isotropic ABPs in a planar Poiseuille flow. Using a generalized Taylor dispersion approach, we derived an effective longitudinal advection-diffusion equation for the cross-sectional average number density. The average drift and effective longitudinal dispersivity are then related to the average and fluctuating field moments, respectively. Our results from this continuum perspective are corroborated by Brownian dynamics simulations of the equations of motion for each active particle. Compared to the BD simulation, the continuum approach exposes the mathematical structure of the problem and elucidates the mechanism from which the observed upstream swimming and dispersive behavior emerge.

Self-propulsion of ABPs leads to interesting behavior in the average drift and effective longitudinal dispersion distinct from those of passive particles. Instead of being advected downstream, ABPs can swim upstream due to the biased rotation from the fluid vorticity. In the absence of flow, fluctuations in self-propulsion alone give rise to enhanced dispersion compared to the translational diffusivity. On the other hand, the combination of flow and activity can lead to a nonmonotonic variation of the effective longitudinal dispersion with changing flow strength. By tuning the relative magnitudes of flow and self-propulsion, the long-time effective dispersivity of ABPs can be minimized.

Both upstream swimming and nonmonotonic dispersion examined in this paper have received considerable attention in previous work using different model swimmers or methods. We have shown that the essential physics required to produce upstream swimming and nonmonotonic 
dispersion are captured by the simple spherical ABP model. Regardless of the type of microorganisms or artificial active particles, the flow effects included in this paper are universally present. As a result, the spherical ABP model provides a basis for the understanding of the transport of active particles in channel flow. In addition, the simplicity of the spherical ABP model allows the development of a continuum theory, which is challenging for active swimmers with complex body geometry.

Several previous works have studied the dynamics of nonspherical particles such as rodlike or beads-spring swimmers. To understand the effect of shape anisotropy, we consider a spheroidal particle and model the orientation dynamics using Jeffery's equation [61]. In the Smoluchowski equation, we need to include the additional effect of alignment from the rate-of-strain tensor $\mathbf{E}$ and write the rotational flux as $\mathbf{j}^{R}=\frac{1}{2} \omega P+B \mathbf{q} \times(\mathbf{E} \cdot \mathbf{q}) P-D_{R} \nabla_{R} P$. When $\mathbf{q}$ is aligned with one of the eigenvectors of $\mathbf{E}$, the dot product $\mathbf{E} \cdot \mathbf{q}$ is parallel to $\mathbf{q}$ and the term $\mathbf{q} \times(\mathbf{E} \cdot \mathbf{q})$ vanishes. The dimensionless parameter $B$ quantifies the shape of the particle. For a sphere, $B=0$. In the strong flow limit, the average drift of a nonspherical particle still approaches that of the passive particles. The effect of the alignment is to enhance upstream swimming when the flow is weak. Because the alignment does not modify the dispersion mechanism, we only expect a weak dependence of the longitudinal dispersion on the shape anisotropy. The effect of particle shape on the average drift and effective longitudinal dispersion is shown in Fig. 15 in Appendix C.

In addition to recovering the upstream swimming and nonmonotonic dispersive behavior discovered by previous work, we systematically examined the effect of the flow speed, the swim speed, the reorientation time, and the translational diffusivity on the dynamics of ABPs in Poiseuille flow. In particular, we studied the dynamics of active particles in the absence of translational diffusion, which has not been analyzed in detail previously. Under weak confinement, active particles without $D_{T}$ experience giant Taylor dispersion where $D^{\text {eff }} / D_{0}^{\text {swim }} \sim \mathrm{Pe}_{\dot{\gamma}}^{4}$ for $\mathrm{Pe}_{\dot{\gamma}} \gg 1$. We showed that a nonmonotonic variation in longitudinal dispersion is also observed in the absence of $D_{T}$ for confined active particles. For ABPs with finite $D_{T}$, we showed that $D^{\text {eff }} / D_{T} \sim \mathrm{Pe}^{2}$ for Pe $\gg 1$. For a fixed $\ell / \delta$ and $\ell / H$, this scaling for finite $D_{T}$ can be alternatively written as $D^{\text {eff }} / D_{0}^{\text {swim }} \sim \mathrm{Pe}_{\dot{\gamma}}^{2}$ using the relation $\mathrm{Pe}_{\dot{\gamma}}=2 \mathrm{Pe}(\ell / H)^{2}(\ell / \delta)^{-2}$. This difference in scaling reveals the singular nature of the limit $D_{T} \rightarrow 0$.

To conclude, we note that wall accumulation and upstream swimming have practical implications such as biofilm formation and bacteria infection. The results we have shown complement our understanding of the dynamics of active particles in Poiseuille flow and provide insights into the effective design of biomedical systems that aim to reduce infection or biofilm formation.

\section{ACKNOWLEDGMENT}

This work is funded by NSF under Grant No. CBET 1803662.

\section{APPENDIX A: GOVERNING EQUATIONS OF THE ORIENTATIONAL MOMENTS}

We substitute the moment expansion of $g_{0}$ and $d_{0}$ into Eqs. (18) and (19) and take orientational moments to obtain the moment equations. The following identities are useful:

$$
\int q_{i} q_{j} d \mathbf{q}=\pi \delta_{i j}, \quad \int q_{i} q_{j} q_{k} q_{l} d \mathbf{q}=\frac{\pi}{4}\left(\delta_{i j} \delta_{k l}+\delta_{i k} \delta_{j l}+\delta_{i l} \delta_{j k}\right),
$$

where $\delta_{i j}$ is the Kronecker delta. At steady state, the average field moments satisfy

$$
\begin{gathered}
\frac{d}{d y}\left(U_{0} m_{y}^{0}-D_{T} \frac{d n^{0}}{d y}\right)=0 \\
\frac{d}{d y}\left(U_{0} Q_{y x}^{0}-D_{T} \frac{d m_{x}^{0}}{d y}\right)+D_{R} m_{x}^{0}+\frac{1}{2} \omega m_{y}^{0}=0
\end{gathered}
$$




$$
\begin{gathered}
\frac{d}{d y}\left(\frac{1}{2} U_{0} n^{0}+U_{0} Q_{y y}^{0}-D_{T} \frac{d m_{y}^{0}}{d y}\right)+D_{R} m_{y}^{0}-\frac{1}{2} \omega m_{x}^{0}=0, \\
\frac{d}{d y}\left(\frac{1}{4} U_{0} m_{y}^{0}-D_{T} \frac{d Q_{y y}^{0}}{d y}\right)+4 D_{R} Q_{y y}^{0}-\omega Q_{y x}^{0}=0 \\
\frac{d}{d y}\left(\frac{1}{4} U_{0} m_{x}^{0}-D_{T} \frac{d Q_{y x}^{0}}{d y}\right)+4 D_{R} Q_{y x}^{0}+\omega Q_{y y}^{0}=0
\end{gathered}
$$

Here, $\omega=8 u_{0} y / H^{2}$ is the scalar vorticity. The no-flux boundary conditions at $y= \pm H / 2$ are given by

$$
\begin{gathered}
U_{0} m_{y}^{0}-D_{T} \frac{d n^{0}}{d y}=0 \\
U_{0} Q_{y x}^{0}-D_{T} \frac{d m_{x}^{0}}{d y}=0, \\
\frac{1}{2} U_{0} n^{0}+U_{0} Q_{y y}^{0}-D_{T} \frac{d}{d y} m_{y}^{0}=0, \\
\frac{1}{4} U_{0} m_{y}^{0}-D_{T} \frac{d Q_{y y}^{0}}{d y}=0, \\
\frac{1}{4} U_{0} m_{x}^{0}-D_{T} \frac{d Q_{y x}^{0}}{d y}=0 .
\end{gathered}
$$

The steady-state moment equations for the fluctuating field are

$$
\begin{gathered}
\frac{d}{d y}\left(U_{0} \tilde{m}_{y}-D_{T} \frac{d \tilde{n}}{d y}\right)=\left(U^{\mathrm{eff}}-u\right) n^{0}-U_{0} m_{x}^{0} \\
\frac{d}{d y}\left(U_{0} \tilde{Q}_{y x}-D_{T} \frac{d \tilde{m}_{x}}{d y}\right)+D_{R} \tilde{m}_{x}+\frac{1}{2} \omega \tilde{m}_{y}=\left(U^{\mathrm{eff}}-u\right) m_{x}^{0}-U_{0}\left(Q_{x x}^{0}+\frac{1}{2} n^{0}\right) \\
\frac{d}{d y}\left(\frac{1}{2} U_{0} \tilde{n}+U_{0} \tilde{Q}_{y y}-D_{T} \frac{d \tilde{m}_{y}}{d y}\right)+D_{R} \tilde{m}_{y}-\frac{1}{2} \omega \tilde{m}_{x}=\left(U^{\mathrm{eff}}-u\right) m_{y}^{0}-U_{0} Q_{y x}^{0}, \\
\frac{d}{d y}\left(\frac{1}{4} U_{0} \tilde{m}_{y}-D_{T} \frac{d \tilde{Q}_{y y}}{d y}\right)+4 D_{R} \tilde{Q}_{y y}-\omega \tilde{Q}_{y x}=\left(U^{\mathrm{eff}}-u\right) Q_{y y}^{0}+\frac{1}{4} U_{0} m_{x}^{0}, \\
\frac{d}{d y}\left(\frac{1}{4} U_{0} \tilde{m}_{x}-D_{T} \frac{d \tilde{Q}_{y x}}{d y}\right)+4 D_{R} \tilde{Q}_{y x}+\omega \tilde{Q}_{y y}=\left(U^{\mathrm{eff}}-u\right) Q_{y x}^{0}-\frac{1}{4} U_{0} m_{y}^{0} .
\end{gathered}
$$

Similar to the average field moments, the fluctuating field moments satisfy the no-flux conditions at the wall:

$$
\begin{gathered}
U_{0} \tilde{m}_{y}-D_{T} \frac{d \tilde{n}}{d y}=0, \\
U_{0} \tilde{Q}_{y x}-D_{T} \frac{d \tilde{m}_{x}}{d y}=0, \\
\frac{1}{2} U_{0} \tilde{n}+U_{0} \tilde{Q}_{y y}-D_{T} \frac{d \tilde{m}_{y}}{d y}=0, \\
\frac{1}{4} U_{0} \tilde{m}_{y}-D_{T} \frac{d \tilde{Q}_{y y}}{d y}=0, \\
\frac{1}{4} U_{0} \tilde{m}_{x}-D_{T} \frac{d \tilde{Q}_{y x}}{d y}=0 .
\end{gathered}
$$


The normalization requires

$$
\frac{1}{H} \int_{-H / 2}^{H / 2} n^{0} d y=1 \text { and } \int_{-H / 2}^{H / 2} \tilde{n} d y=0 .
$$

We solve these equations in MATLAB using a Chebyshev collocation method [62]. Equation (A2) along with its boundary condition (A7) is analytically integrated once to obtain a first-order equation:

$$
U_{0} m_{y}^{0}-D_{T} \frac{d n^{0}}{d y}=0 .
$$

To solve the average field moment equations (A3)-(A6) and (A23), we start from a guess for $n^{0}(-H / 2)$ as a boundary condition and update the guess based on a root finding algorithm such that the normalization condition is satisfied within a tolerance of $10^{-6}$. A similar approach for the fluctuating field equations is used.

\section{APPENDIX B: BROWNIAN DYNAMICS SIMULATION}

The motion of ABPs is governed by the overdamped Langevin equations as presented in Eqs. (24) and (25). In two dimensions, we can parametrize the orientation vector by an orientation angle $\theta$ such that $\mathbf{q}=\cos \theta \mathbf{e}_{x}+\sin \theta \mathbf{e}_{y}$. The discretized Langevin equation is given by

$$
\begin{aligned}
\Delta x & =u\left(y_{n}\right) \Delta t+U_{0} \cos \left[\theta\left(t_{n}\right)\right] \Delta t+\Delta x^{B}, \\
\Delta y & =U_{0} \sin \left[\theta\left(t_{n}\right)\right] \Delta t+\Delta y^{B}, \\
\Delta \theta & =\frac{1}{2} \omega\left(y_{n}\right) \Delta t+\Delta \theta^{B},
\end{aligned}
$$

where $\Delta x$ is the particle displacement in the $x$ direction over the time step $\Delta t$ from $t_{n}$ to $t_{n+1}$. Similarly, $\Delta y$ is the displacement in the $y$ direction and $\Delta \theta$ is the angular displacement. The Brownian displacement $\Delta x^{B}$ (or $\Delta y^{B}$ ) is sampled from a white noise with a mean of zero and variance of $2 D_{T} \Delta t$. Similarly, the rotary Brownian displacement $\Delta \theta^{B}$ has a mean of zero and variance of $2 \Delta t / \tau_{R}$. The subscript $n$ denotes the current time, and $t_{n+1}=t_{n}+\Delta t$. The position and orientation of a particle at $t_{n+1}$ are then given by $x_{n+1}=x_{n}+\Delta x, y_{n+1}=y_{n}+\Delta y, \theta_{n+1}=\theta_{n}+\Delta \theta$. The position $y_{n+1}$ is then corrected according to the potential-free algorithm if it overlaps with the channel wall. In our definition, the parameter $H$ already includes the radius of the particle. In other words, when the center position of the particles exceeds $\pm H / 2$, we move the particle back such that its center position is at $\pm H / 2$. The particle size does not appear explicitly in the potential-free algorithm. We note that the radius of the ABPs is assumed to be negligible compared to the width of the channel. As a result, the ABPs experience zero background flow at contact with the wall.

In most simulations, we use a time step $\Delta t=10^{-3} t_{\min }$ where $t_{\min }$ is the smallest time scale in the problem, which is estimated via $t_{\min }=\min \left(\tau_{R}, H^{2} / D_{T}, H / U_{0}, H / u_{0}\right)$. Simulations were run for a duration of $100 t_{\max }$ where $t_{\max }$ is the largest time scale in the problem. All simulations are performed using an in-house GPU-accelerated code consisting of 102400 active Brownian particles.

\section{APPENDIX C: AVERAGE DRIFT AND LONGITUDINAL DISPERSION OF NONSPHERICAL PARTICLES}

In Fig. 15(a), we show the average drift as a function of Pe for different values of $B$. For an ellipsoidal particle of major axis $a$ and minor axis $b$, the shape factor is defined as $B=$ $\left(r^{2}-1\right) /\left(r^{2}+1\right)$, where the aspect ratio $r=a / b$. For a sphere $(a=b)$, we have $B=0$. For a rodlike particle, $r \rightarrow \infty$ and $B \rightarrow 1$. As $B$ increases from 0 to 1 , the particle shape changes from a sphere to a rod. In the weak flow limit, alignment from the rate-of-strain tensor enhances upstream swimming of nonspherical particles. In Fig. 15(b), we show the effective longitudinal dispersion coefficient as a function of Pe for different values of $B$. We only observe a weak dependence of the 

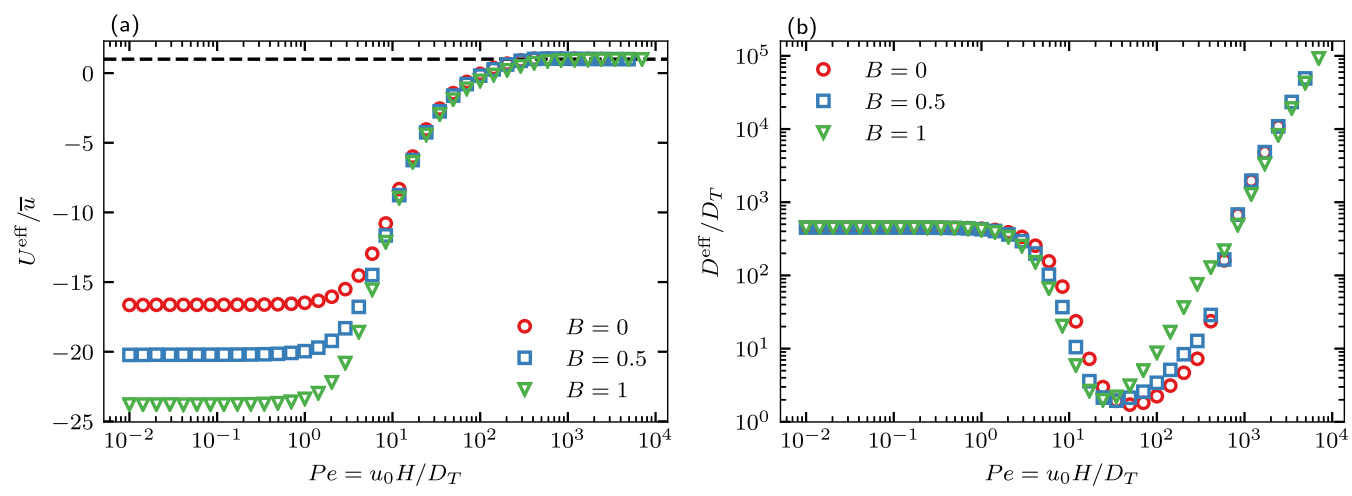

FIG. 15. (a) The average drift $U^{\text {eff }} / \bar{u}$ as a function of Pe for different values of $B$. The black dashed line is the result for passive Brownian particles, $U^{\text {eff }} / \bar{u} \equiv 1$. (b) The effective longitudinal dispersion coefficient $D^{\text {eff }} / D_{T}$ as a function of Pe for different values of $B$. In both panels, $\ell / H=10$ and $\ell / \delta=30$. Results are obtained from FEM simulation of the Smoluchowski equation.

dispersion on particle shape. We note that the same no-flux boundary condition for spherical ABPs $\left(\mathbf{e}_{y} \cdot \mathbf{j}^{T}=0\right.$ at the wall) is used in the Smoluchowski equation for nonspherical particles.

[1] A. Siitonen and M. Nurminen, Bacterial motility is a colonization factor in experimental urinary tract infection, Infect. Immun. 60, 3918 (1992).

[2] C. Y. Kao, W. H. Lin, C. C. Tseng, A. B. Wu, M. C. Wang, and J. J. Wu, The complex interplay among bacterial motility and virulence factors in different escherichia coli infections, Eur. J. Clin. Microbiol. Infect. Dis. 33, 2157 (2014).

[3] R. Rusconi, S. Lecuyer, L. Guglielmini, and H. A. Stone, Laminar flow around corners triggers the formation of biofilm streamers, J. R. Soc., Interface 7, 1293 (2010).

[4] M. K. Kim, K. Drescher, O. S. Pak, B. L. Bassler, and H. A. Stone, Filaments in curved streamlines: Rapid formation of Staphylococcus aureus biofilm streamers, New J. Phys. 16, 065024 (2014).

[5] C. Bechinger, R. Di Leonardo, H. Löwen, C. Reichhardt, G. Volpe, and G. Volpe, Active particles in complex and crowded environments, Rev. Mod. Phys. 88, 045006 (2016).

[6] G. I. Taylor, Dispersion of soluble matter in solvent flowing slowly through a tube, Proc. R. Soc. A 219, 186 (1953).

[7] G. I. Taylor, The dispersion of matter in turbulent flow through a pipe, Proc. R. Soc. A 223, 446 (1954).

[8] G. I. Taylor, Conditions under which dispersion of a solute in a stream of solvent can be used to measure molecular diffusion, Proc. R. Soc. A 225, 473 (1954).

[9] R. Aris, On the dispersion of a solute in a fluid flowing through a tube, Proc. R. Soc. A 235, 67 (1956).

[10] H. Brenner, Dispersion resulting from flow through spatially periodic porous media, Philos. Trans. R. Soc. A 297, 81 (1980).

[11] K. D. Dorfman and H. Brenner, Generalized Taylor-Aris dispersion in discrete spatially periodic networks: Microfluidic applications, Phys. Rev. E 65, 021103 (2002).

[12] M. Shapiro and H. Brenner, Taylor dispersion of chemically reactive species: Irreversible first-order reactions in bulk and on boundaries, Chem. Eng. Sci. 41, 1417 (1986).

[13] M. Shapiro and H. Brenner, Chemically reactive generalized Taylor dispersion phenomena, AIChE J. 33, 1155 (1987).

[14] I. Frankel and H. Brenner, On the foundations of generalized Taylor dispersion theory, J. Fluid Mech. 204, 97 (1989).

[15] H. Brenner and A. D. Edwards, Macrotransport Processes (Elsevier, Amsterdam, 2013). 
[16] H. C Berg, Random Walks in Biology (Princeton University, Princeton, NJ, 1993).

[17] S. C. Takatori, W. Yan, and J. F. Brady, Swim Pressure: Stress Generation in Active Matter, Phys. Rev. Lett. 113, 028103 (2014).

[18] S. C. Takatori and J. F. Brady, Swim stress, motion, and deformation of active matter: Effect of an external field, Soft Matter 10, 9433 (2014).

[19] A. Sokolov, M. M. Apodaca, B. A. Grzybowski, and I. S. Aranson, Swimming bacteria power microscopic gears, Proc. Natl. Acad. Sci. USA 107, 969 (2010).

[20] L. Rothschild, Non-random distribution of bull spermatozoa in a drop of sperm suspension, Nature (London) 200, 381 (1963).

[21] A. P. Berke, L. Turner, H. C. Berg, and E. Lauga, Hydrodynamic Attraction of Swimming Microorganisms by Surfaces, Phys. Rev. Lett. 101, 038102 (2008).

[22] G. Li and J. X. Tang, Accumulation of Microswimmers Near a Surface Mediated by Collision and Rotational Brownian Motion, Phys. Rev. Lett. 103, 078101 (2009).

[23] G. Li, J. Bensson, L. Nisimova, D. Munger, P. Mahautmr, J. X. Tang, M. R. Maxey, and Y. V. Brun, Accumulation of swimming bacteria near a solid surface, Phys. Rev. E 84, 041932 (2011).

[24] A. Costanzo, R. D. Leonardo, G. Ruocco, and L. Angelani, Transport of self-propelling bacteria in microchannel flow, J. Phys.: Condens. Matter 24, 065101 (2012).

[25] J. Elgeti and G. Gompper, Wall accumulation of self-propelled spheres, Europhys. Lett. 101, 48003 (2013).

[26] W. Yan and J. F. Brady, The swim force as a body force, Soft Matter 11, 6235 (2015).

[27] W. Yan and J. F. Brady, The curved kinetic boundary layer of active matter, Soft Matter 14, 279 (2018).

[28] W. Yan and J. F. Brady, The force on a boundary in active matter, J. Fluid Mech. 785, R1 (2015).

[29] J. Hill, O. Kalkanci, J. L. McMurry, and H. Koser, Hydrodynamic Surface Interactions Enable Escherichia Coli to Seek Efficient Routes to Swim Upstream, Phys. Rev. Lett. 98, 068101 (2007).

[30] T. Kaya and H. Koser, Characterization of Hydrodynamic Surface Interactions of Escherichia Coli Cell Bodies in Shear Flow, Phys. Rev. Lett. 103, 138103 (2009).

[31] T. Kaya and H. Koser, Direct upstream motility in Escherichia coli, Biophys. J. 102, 1514 (2012).

[32] V. Kantsler, J. Dunkel, M. Blayney, and R. E Goldstein, Rheotaxis facilitates upstream navigation of mammalian sperm cells, Elife 3, e02403 (2014).

[33] R. W. Nash, R. Adhikari, J. Tailleur, and M. E. Cates, Run-and-Tumble Particles with Hydrodynamics: Sedimentation, Trapping, and Upstream Swimming, Phys. Rev. Lett. 104, 258101 (2010).

[34] A. Zöttl and H. Stark, Nonlinear Dynamics of A Microswimmer in Poiseuille Flow, Phys. Rev. Lett. 108, 218104 (2012).

[35] W. E. Uspal, M. N. Popescu, S. Dietrich, and M. Tasinkevych, Self-propulsion of a catalytically active particle near a planar wall: From reflection to sliding and hovering, Soft Matter 11, 434 (2015).

[36] B. Ezhilan and D. Saintillan, Transport of a dilute active suspension in pressure-driven channel flow, J. Fluid Mech. 777, 482 (2015).

[37] G. Junot, N. Figueroa-Morales, T. Darnige, A. Lindner, R. Soto, H. Auradou, and E. Clément, Swimming bacteria in poiseuille flow: The quest for active Bretherton-Jeffery trajectories, Europhy. Lett. 126, 44003 (2019).

[38] A. J. T. M. Mathijssen, N. Figueroa-Morales, G. Junot, É. Clément, A. Lindner, and A. Zöttl, Oscillatory surface rheotaxis of swimming E. coli bacteria, Nat. Commun. 10, 3434 (2019).

[39] T. J. Pedley and J. O. Kessler, A new continuum model for suspensions of gyrotactic micro-organisms, J. Fluid Mech. 212, 155 (1990).

[40] N. A. Hill and M. A. Bees, Taylor dispersion of gyrotactic swimming micro-organisms in a linear flow, Phys. Fluids 14, 2598 (2002).

[41] A. Manela and I. Frankel, Generalized Taylor dispersion in suspensions of gyrotactic swimming microorganisms, J. Fluid Mech. 490, 99 (2003).

[42] M. A. Bees and O. A. Croze, Dispersion of biased swimming micro-organisms in a fluid flowing through a tube, Proc. R. Soc. A 466, 2057 (2010).

[43] O. A. Croze, G. Sardina, M. Ahmed, M. A. Bees, and L. Brandt, Dispersion of swimming algae in laminar and turbulent channel flows: consequences for photobioreactors, J. R. Soc. Interface 10, 20121041 (2013). 
[44] R. N. Bearon, A. L. Hazel, and G. J. Thorn, The spatial distribution of gyrotactic swimming microorganisms in laminar flow fields, J. Fluid Mech. 680, 602 (2011).

[45] R. N. Bearon, An extension of generalized Taylor dispersion in unbounded homogeneous shear flows to run-and-tumble chemotactic bacteria, Phys. Fluids 15, 1552 (2003).

[46] S. Chilukuri, C. H. Collins, and P. T. Underhill, Dispersion of flagellated swimming microorganisms in planar Poiseuille flow, Phys. Fluids 27, 031902 (2015).

[47] R. Alonso-Matilla, B. Chakrabarti, and D. Saintillan, Transport and dispersion of active particles in periodic porous media, Phys. Rev. Fluids 4, 043101 (2019).

[48] A. Dehkharghani, N. Waisbord, J. Dunkel, and J. S. Guasto, Bacterial scattering in microfluidic crystal flows reveals giant active Taylor-Aris dispersion, Proc. Natl. Acad. Sci. USA 116, 11119 (2019).

[49] W. Jiang and G. Chen, Dispersion of active particles in confined unidirectional flows, J. Fluid Mech. 877, 1 (2019).

[50] D. Saintillan and M. J. Shelley, Theory of active suspensions, in Complex Fluids in Biological Systems: Experiment, Theory, and Computation, edited by Saverio E. Spagnolie (Springer, New York, 2015), pp. 319-355.

[51] R. N. Zia and J. F. Brady, Single-particle motion in colloids: Force-induced diffusion, J. Fluid Mech. 658, 188 (2010).

[52] D. Saintillan and M. J. Shelley, Active suspensions and their nonlinear models, C. R. Phys. 14, 497 (2013).

[53] M. Theillard, R. Alonso-Matilla, and D. Saintillan, Geometric control of active collective motion, Soft Matter 13, 363 (2017).

[54] F. Hecht, New development in freefem++, J. Numer. Math. 20, 251 (2012).

[55] D. M. Heyes and J. R. Melrose, Brownian dynamics simulations of model hard-sphere suspensions, J. Non-Newtonian Fluid Mech. 46, 1 (1993).

[56] Marcos, H. C. Fu, T. R. Powers, and R. Stocker, Bacterial rheotaxis, Proc. Natl. Acad. Sci. USA 109, 4780 (2012).

[57] Y. Shen, A. Siryaporn, S. Lecuyer, Z. Gitai, and H. A. Stone, Flow directs surface-attached bacteria to twitch upstream, Biophys. J. 103, 146 (2012).

[58] Chih-kuan Tung, F. Ardon, A. Roy, D. L. Koch, S. S. Suarez, and M. Wu, Emergence of Upstream Swimming Via A Hydrodynamic Transition, Phys. Rev. Lett. 114, 108102 (2015).

[59] T. Omori and T. Ishikawa, Upward swimming of a sperm cell in shear flow, Phys. Rev. E 93, 032402 (2016).

[60] S. Chilukuri, C. H. Collins, and P. T. Underhill, Impact of external flow on the dynamics of swimming microorganisms near surfaces, J. Phys.: Condens. Matter 26, 115101 (2014).

[61] G. B. Jeffery and L. N. G. Filon, The motion of ellipsoidal particles immersed in a viscous fluid, Proc. R. Soc. A 102, 161 (1922).

[62] L. N. Trefethen, Spectral Methods in MATLAB (SIAM, New York, 2000), Vol. 10. 